TRANSACTIONS OF THE

AMERICAN MATHEMATICAL SOCIETY

Volume 358, Number 1, Pages 441-472

S 0002-9947(05)03774-8

Article electronically published on August 1, 2005

\title{
A DENSITY THEOREM ON AUTOMORPHIC $L$-FUNCTIONS AND SOME APPLICATIONS
}

\author{
YUK-KAM LAU AND JIE WU
}

\begin{abstract}
We establish a density theorem on automorphic $L$-functions and give some applications on the extreme values of these $L$-functions at $s=1$ and the distribution of the Hecke eigenvalue of holomorphic cusp forms.
\end{abstract}

\section{INTRODUCTION}

The distribution of zeros is an important area of the study of $L$-functions. There are many arithmetical problems related to the location of the zeros. It is widely believed that the generalized Riemann hypothesis (GRH) holds, but a proof for this seems out of reach at present. In the absence of GRH, the zero density estimates are often used as a substitute in many applications, especially for the number of possible zeros close to the boundary of the critical strip. The result of such an estimate is called a density theorem. The first zero density result for the symmetric square $L$-function of Maass forms with large eigenvalues was obtained by Luo [15]. Very recently Kowalski \& Michel [13] have proved a very general density theorem for automorphic $L$-functions with large conductors, which includes the case of holomorphic cusp forms for large levels.

In this paper, we shall consider the analogue on the weight aspect. Our work is motivated by two factors. First it is natural to investigate the behaviour of an automorphic $L$-function by varying each intrinsic parameter. Second we are interested in the following applications: the extreme values of automorphic $L$-functions at $s=1$ and the distribution of Fourier coefficients of holomorphic cusp forms.

Let us begin with our notation. For a positive even integer $k$ and a positive square-free integer $N$, we denote by $\mathrm{H}_{k}^{*}(N)$ the set of all normalized Hecke primitive eigencuspforms of weight $k$ for the congruence modular group $\Gamma_{0}(N)$. Then, $\mathrm{H}_{k}^{*}(N)$ forms an orthogonal basis of the space of holomorphic cuspidal newforms (of weight $k$ and of level $N$ ). We have

$$
\left|\mathrm{H}_{k}^{*}(N)\right|=\frac{k-1}{12} \prod_{p \mid N}(p-1)+O\left((k N)^{2 / 3}\right),
$$

where the implied constant is absolute.

Received by the editors November 8, 2003 and, in revised form, June 23, 2004.

2000 Mathematics Subject Classification. Primary 11F67, 11F30.

Key words and phrases. Special values of automorphic $L$-series, Fourier coefficients of automorphic forms.

(C)2005 American Mathematical Society Reverts to public domain 28 years from publication 
The Fourier series expansion of $f \in \mathrm{H}_{k}^{*}(N)$ at the cusp $\infty$ is

$$
f(z)=\sum_{n=1}^{\infty} \lambda_{f}(n) n^{(k-1) / 2} e^{2 \pi i n z} \quad(\Im m z>0),
$$

where $\lambda_{f}(n)$ is the $n$-th eigenvalue of the (normalized) Hecke operator $T_{n}$, in particular it is a multiplicative function of $n$. According to Deligne, for any prime number $p$ there are $\alpha_{f}(p)$ and $\beta_{f}(p)$ such that

$$
\lambda_{f}\left(p^{\nu}\right)=\frac{\alpha_{f}(p)^{\nu+1}-\beta_{f}(p)^{\nu+1}}{\alpha_{f}(p)-\beta_{f}(p)} \quad(\nu \geq 1)
$$

and

$$
\begin{cases}\alpha_{f}(p)=\varepsilon_{f}(p) p^{-1 / 2}, \beta_{f}(p)=0 & \text { if } p \mid N, \\ \left|\alpha_{f}(p)\right|=\alpha_{f}(p) \beta_{f}(p)=1 & \text { if } p \nmid N\end{cases}
$$

with $\varepsilon_{f}(p)= \pm 1$. In particular $\lambda_{f}(1)=1$ and $\lambda_{f}(n)$ is real.

The $m$-th symmetric power $L$-function attached to $f \in \mathrm{H}_{k}^{*}(N)$ is defined as

$$
L\left(s, \operatorname{sym}^{m} f\right):=\prod_{p} \prod_{0 \leq j \leq m}\left(1-\alpha_{f}(p)^{m-j} \beta_{f}(p)^{j} p^{-s}\right)^{-1}
$$

for $\sigma>1$, where and in the sequel $\sigma$ and $\tau$ mean tacitly the real and imaginary part of $s$, i.e. $s=\sigma+i \tau$. The product over primes admits a Dirichlet series representation: for $\sigma>1$,

$$
L\left(s, \operatorname{sym}^{m} f\right)=\sum_{n=1}^{\infty} \lambda_{\text {sym }^{m}}(n) n^{-s},
$$

where $\lambda_{\text {sym }}{ }_{f}(n)$ is a multiplicative function. Following from (1.3) and (1.4), we have for $n \geq 1$,

$$
\left|\lambda_{\text {sym }^{m} f}(n)\right| \leq \mathrm{d}_{m+1}(n),
$$

where $\mathrm{d}_{m+1}(n)$ is the divisor function whose associated Dirichlet series is $\zeta(s)^{m+1}$ $(\zeta(s)$ is the Riemann zeta-function). The case $m=1$ in (1.6) is commonly known as Deligne's inequality.

According to [1, Section 3.2.1], the gamma factors of $L\left(s, \operatorname{sym}^{m} f\right)$ are

$$
L_{\infty}\left(s, \operatorname{sym}^{m} f\right):= \begin{cases}\prod_{\nu=0}^{n} \Gamma_{\mathbb{C}}\left(s+\left(\nu+\frac{1}{2}\right)(k-1)\right) & \text { if } m=2 n+1, \\ \Gamma_{\mathbb{R}}\left(s+\delta_{2 \nmid n}\right) \prod_{\nu=1}^{n} \Gamma_{\mathbb{C}}(s+\nu(k-1)) & \text { if } m=2 n,\end{cases}
$$

where $\Gamma_{\mathbb{R}}(s):=\pi^{-s / 2} \Gamma(s / 2), \Gamma_{\mathbb{C}}(s):=2(2 \pi)^{-s} \Gamma(s)$ and

$$
\delta_{2 \nmid n}:= \begin{cases}1 & \text { if } 2 \nmid n, \\ 0 & \text { otherwise. }\end{cases}
$$

For $m=1,2,3,4$, it is known (see [7] for $m=1$, [2] for $m=2$ and [9, 10, 11] for $m=3,4)$ that the function

$$
\Lambda\left(s, \operatorname{sym}^{m} f\right):=N^{m s / 2} L_{\infty}\left(s, \operatorname{sym}^{m} f\right) L\left(s, \operatorname{sym}^{m} f\right)
$$


is entire on $\mathbb{C}$ and satisfies the functional equation

$$
\Lambda\left(s, \operatorname{sym}^{m} f\right)=\varepsilon_{\text {sym }^{m}} \Lambda\left(1-s, \operatorname{sym}^{m} f\right),
$$

where $\varepsilon_{\mathrm{sym}^{m} f}= \pm 1$.

1.1. The density theorem for $L\left(s, \operatorname{sym}^{m} f\right)$. We consider the possibility of the existence of a zero $\rho=\beta+i \gamma$ of $L\left(s, \operatorname{sym}^{m} f\right)$ for which $\beta$ is near 1 . It is hopeful to show that such $f \in \mathrm{H}_{k}^{*}(1)$ are very few. In other words, "almost all" $f \in \mathrm{H}_{k}^{*}(1)$ satisfy the quasi-hypothesis of Riemann. Let $N\left(\alpha, T, \operatorname{sym}^{m} f\right)$ be the number of zeros $\rho=\beta+i \gamma$ of $L\left(s, \operatorname{sym}^{m} f\right)$ with $\beta \geq \alpha$ and $0 \leq \gamma \leq T$.

Our result is as follows.

Theorem 1. Let $m=1,2,3,4$ and $r \geq 1$ be given. Define $E_{m, r}=(m+1)(m+r)+4$. Then for any $\varepsilon>0$, we have

$$
\sum_{f \in \mathrm{H}_{k}^{*}(1)} N\left(\alpha, T, \operatorname{sym}^{m} f\right) \ll_{\varepsilon, r} T^{1+1 / r} k^{E_{m, r}(1-\alpha) /(3-2 \alpha)+\varepsilon}
$$

uniformly for $\frac{1}{2}+\varepsilon \leq \alpha \leq 1,2 \mid k$ and $T \geq 1$. The implied constant depends on $\varepsilon$ and $r$ only.

Remark 1. (i) This theorem is nontrivial only when $\alpha$ is very close to 1 and the $T$-aspect is essentially irrelevant. We have not put any effort into reducing the exponents $1+1 / r$ and $E_{m, r}$.

(ii) Since we are interested in the $k$-aspect, we restrict ourselves to the case $N=1$ for simplicity. All results of this paper can be generalized (without too much difficulty) to $\mathrm{H}_{k}^{*}(N)$ with square-free $N$.

(iii) Theorem 1 is established only for the case $1 \leq m \leq 4$ due to the lack of knowledge about the high symmetric powers. One can extend the result to the general case for all positive integers $m$ under suitable assumptions (Hypothesis $\left.\mathrm{Sym}^{m}(f)\right)$. Interested readers are referred to [1] for an excellent paradigm.

For each $\eta \in\left(0, \frac{1}{2}\right)$, define

$$
\mathrm{H}_{k, \mathrm{sym}^{m}}^{+}(1 ; \eta):=\left\{f \in \mathrm{H}_{k}^{*}(1): L\left(s, \operatorname{sym}^{m} f\right) \neq 0, s \in \mathcal{S}\right\},
$$

where $\mathcal{S}:=\left\{s: \sigma \geq 1-\eta,|\tau| \leq 100 k^{\eta}\right\} \cup\{s: \sigma \geq 1\}$, and

$$
\mathrm{H}_{k, \text { sym }^{m}}^{-}(1 ; \eta):=\mathrm{H}_{k}^{*}(1) \backslash \mathrm{H}_{k, \text { sym }^{m}}^{+}(1 ; \eta) .
$$

Then an immediate consequence of Theorem 1 (with $r=1$ ) is

$$
\begin{aligned}
\left|\mathrm{H}_{k, \mathrm{sym}^{m}}^{-}(1 ; \eta)\right| & \leq \sum_{f \in \mathrm{H}_{k, \mathrm{sym}}^{-}(1 ; \eta)} N\left(1-\eta, 100 k^{\eta}, \operatorname{sym}^{m} f\right) \\
& \leq \sum_{f \in \mathrm{H}_{k}^{*}(1)} N\left(1-\eta, 100 k^{\eta}, \operatorname{sym}^{m} f\right) \\
& \ll_{\eta} k^{31 \eta} .
\end{aligned}
$$

Combining this with (1.1), we obtain the following result.

Corollary 1. Let $2 \mid k$, let $m=1,2,3,4$ and let $\eta \in\left(0, \frac{1}{31}\right)$. Then we have, for $k \rightarrow \infty$,

$$
\left|\mathrm{H}_{k, \mathrm{sym}^{m}}^{+}(1 ; \eta)\right| \sim\left|\mathrm{H}_{k}^{*}(1)\right| \text {. }
$$


This shows that for $m=1,2,3,4$, the functions $L\left(s, \operatorname{sym}^{m} f\right)$ of almost all $f \in$ $\mathrm{H}_{k}^{*}(1)$ satisfy a weak form of GRH. Thus the zero density result is very useful and often partially replaces the role of GRH in practice. As opposed to previous works (see [18, [19], 20], 21], 5] and [1]), we shall consider $\mathrm{H}_{k, \mathrm{sym}^{m}}^{+}(1 ; \eta)$ instead of $\mathrm{H}_{k}^{*}(1)$. An advantage of this choice is that we can avoid some assumptions there (such as GRH in [21] and Cogdell \& Michel's hypothesis $\operatorname{LSZ}^{m}(N)$ in [1]). Next we shall present some applications of Theorem 1 and Corollary 1 .

1.2. Extreme values of $L\left(1, \operatorname{sym}^{m} f\right)$. Motivated by problems in spectral deformation theory, Luo [15] studied the distribution of the values of the symmetric square $L$-functions of Maass forms at $s=1$. Luo's work was extended and further developed in [18, [19], 20, 21, [5] and [1, where the symmetric power $L$-functions attached to holomorphic cusp forms with large square-free levels were investigated. Here we are interested in the extreme values of $L\left(1, \operatorname{sym}^{m} f\right)$ on the weight aspect.

The Hoffstein-Lockhart bounds for $L\left(1, \operatorname{sym}^{m} f\right)$ are (see [6] and [3])

$$
[\log (k N)]^{-1} \ll L\left(1, \operatorname{sym}^{m} f\right) \ll \log (k N)
$$

for all $f \in \mathrm{H}_{k}^{*}(N)$ and $m=1,2$, where the implied constants are absolute. When $m \geq 3$, the relevant results can be found in [1] on the level aspect and in Proposition 3.2 below on the weight aspect for $N=1$. The order of magnitudes of both the upper and lower bounds are (respectively positive and negative) powers of $\log k$.

We prove the following result in the opposite direction. As usual, we denote by $\log _{j}$ the $j$-fold iterated logarithm.

Theorem 2. Let $\eta \in\left(0, \frac{1}{31}\right)$ be fixed, let $m=1,2,3,4$ and let $2 \mid k$. Then there are $f_{m}^{ \pm} \in \mathrm{H}_{k, \mathrm{sym}^{m}}^{+}(1 ; \eta)$ such that, for $k \rightarrow \infty$,

$$
\begin{aligned}
& L\left(1, \operatorname{sym}^{m} f_{m}^{+}\right) \geq\{1+o(1)\}\left(B_{m}^{+} \log _{2} k\right)^{A_{m}^{+}}, \\
& L\left(1, \operatorname{sym}^{m} f_{m}^{-}\right) \leq\{1+o(1)\}\left(B_{m}^{-} \log _{2} k\right)^{-A_{m}^{-}},
\end{aligned}
$$

where $A_{m}^{ \pm}$and $B_{m}^{ \pm}$are positive constants given by

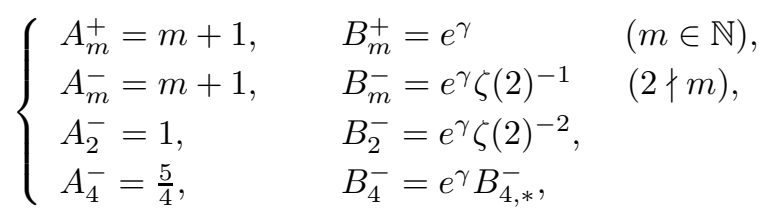

and

$$
\begin{aligned}
B_{4, *}^{-}= & \prod_{p}\left\{( 1 - \frac { 1 } { p } ) \left[\frac{2 p}{27}\left(-1+\frac{10}{p}+\frac{6}{p^{2}}+\frac{3}{p^{3}}-\frac{3}{p^{4}}-\frac{6}{p^{5}}-\frac{10}{p^{6}}+\frac{1}{p^{7}}\right)\right.\right. \\
& \left.\left.+\frac{2 p}{27}\left(1+\frac{2}{p}+\frac{5}{p^{2}}-\frac{5}{p^{3}}-\frac{2}{p^{4}}-\frac{1}{p^{5}}\right) \sqrt{1+\frac{3}{p}+\frac{8}{p^{2}}+\frac{3}{p^{3}}+\frac{1}{p^{4}}}\right]^{4 / 5}\right\} .
\end{aligned}
$$

Here $\gamma$ is Euler's constant.

There are wide gaps between the results mentioned in (1.12) and those in Theorem 2, the former is of size powers of $\log k$ while the latter is of powers of $\log _{2} k$. Our next result suggests that the latter estimates should be closer to the truth. 
For almost all $f \in \mathrm{H}_{k}^{*}(1)$, the magnitude of $L\left(1, \operatorname{sym}^{m} f\right)$ lies between the powers of $\log _{2} k$ shown in (1.13) and (1.14), so Theorem 2 is the best possible up to a constant factor. To determine the plausible constants, we consider the conditional result under GRH. The constants obtained turn out to be quite near those in Theorem 2

Theorem 3. Let $m=1,2,3,4$ and let $2 \mid k$.

(i) For any fixed $\eta \in\left(0, \frac{1}{31}\right)$ and all $f \in \mathrm{H}_{k, \mathrm{sym}^{m}}^{+}(1 ; \eta)$, we have

$$
\left(\log _{2} k\right)^{-A_{m}^{-}} \ll L\left(1, \operatorname{sym}^{m} f\right) \ll\left(\log _{2} k\right)^{A_{m}^{+}} .
$$

(ii) For any $f \in \mathrm{H}_{k}^{*}(1)$, under $G R H$ for $L\left(s, \operatorname{sym}^{m} f\right)$ we have, for $k \rightarrow \infty$,

$$
\{1+o(1)\}\left(2 B_{m}^{-} \log _{2} k\right)^{-A_{m}^{-}} \leq L\left(1, \operatorname{sym}^{m} f\right) \leq\{1+o(1)\}\left(2 B_{m}^{+} \log _{2} k\right)^{A_{m}^{+}} .
$$

The constants $A_{m}^{ \pm}$and $B_{m}^{ \pm}$are defined as in (1.15) and (1.16).

Remark 2. (i) In the extreme value problem of $L\left(1, \mathrm{sym}^{m} f\right)$, the result on the weight aspect is different from that on the level aspect. As proved in 20] and 21, the extreme values of $L\left(1, \operatorname{sym}^{m} f\right)$ are attained only for special levels (free of small prime factors).

(ii) Only the factor 2 in (1.18) remains in doubt on either side, in view of (1.13) and (1.14).

1.3. Asymptotic distributions of $\lambda_{f}(p)$. The distribution of Fourier coefficients of modular forms is one of the most important problems in the theory of modular forms. Various questions are raised and studied: upper bound estimate, equidistribution property, lacunarity, etc. Let $\tau(n)$ be Ramanujan's function, defined by

$$
\Delta(z):=e^{2 \pi i z} \prod_{n=1}^{\infty}\left(1-e^{2 \pi i n z}\right)^{12}=\sum_{n=1}^{\infty} \tau(n) e^{2 \pi i n z} \quad(\Im m z>0) .
$$

The function $\Delta(z)$ is a holomorphic cusp form of weight 12 , i.e. $\Delta(z) \in \mathrm{H}_{12}^{*}(1)$. The classical Ramanujan's conjecture states

$$
|\tau(n)| \leq \mathrm{d}(n) n^{11 / 2} \quad(n \geq 1),
$$

which is essentially optimal since Rankin [17] showed that

$$
\limsup _{n \rightarrow \infty} \frac{\tau(n)}{n^{11 / 2}}=\infty .
$$

Ramanujan's conjecture was proved by Deligne in 1974 as a particular case of his well-known inequality (1.6). In particular this inequality gives us

$$
\left|\lambda_{f}(p)\right| \leq 2
$$

for all $f \in \mathrm{H}_{k}^{*}(N)$ and all prime number $p$. Serre [24, page 81] showed that this inequality is essentially optimal: for any fixed prime number $p$ and for any $\varepsilon>0$, there is a constant $x_{0}=x_{0}(\varepsilon)$ such that for $k+N \geq x_{0}$ with $p \nmid N$ there is a primitive form $f \in \mathrm{H}_{k}^{*}(N)$ having the following property:

$$
\left|\lambda_{f}(p)\right| \geq 2-\varepsilon .
$$

Later he proved that (1.22) holds for any fixed finite set of prime numbers ([24, page 87]). Very recently, under GRH for $L\left(s, \operatorname{sym}^{1} f\right)$, Royer and Wu [21] extended it to the case of the unbounded set of primes for some primitive forms of sufficiently large levels. 
Here we shall establish an analogue of [21, corollaire $\mathrm{H}]$ for large weights, but the assumption of GRH is removed!

Theorem 4. Let $A>0$, let $\eta \in\left(0, \frac{1}{31}\right)$ fixed, let $2 \mid k$ and let $\xi(k) \rightarrow \infty(k \rightarrow \infty)$ be a function satisfying $\xi(k) \leq \log _{3} k$. Then there are two forms $f_{ \pm} \in \mathrm{H}_{k, \mathrm{sym}^{1}}^{+}(1 ; \eta)$ such that, for $k \rightarrow \infty$,

$$
\begin{gathered}
\sum_{\substack{p \leq(\log k)^{A} \\
\lambda_{f_{+}}(p) \geq 2-\xi(k) / \log _{3} k}} \frac{1}{p}=\left(\log _{3} k\right)\left\{1+O_{A, \eta}\left(\frac{1}{\xi(k)}\right)\right\}, \\
\sum_{\substack{p \leq(\log k)^{A} \\
\lambda_{f_{-}}(p) \leq-2+\xi(k) / \log _{3} k}} \frac{1}{p}=\left(\log _{3} k\right)\left\{1+O_{A, \eta}\left(\frac{1}{\xi(k)}\right)\right\},
\end{gathered}
$$

respectively.

Remark 3. The well-known Sato-Tate conjecture describes the distribution of the Fourier coefficients, as follows: for any $-2 \leq \alpha \leq \beta \leq 2$ and any $f \in \mathrm{H}_{k}^{*}(N)$, one has

$$
\left|\left\{p \leq x: \alpha \leq \lambda_{f}(p) \leq \beta\right\}\right| \sim \frac{x}{\log x} \int_{\alpha}^{\beta} \frac{\sqrt{4-t^{2}}}{2 \pi} \mathrm{d} t \quad(x \rightarrow \infty) .
$$

Theorem 4 shows that almost all initial terms of $\left\{\lambda_{f}(p)\right\}_{p}$ cluster around 2 or -2 . Hence Sato-Tate's conjecture is not yet valid for $x=(\log k)^{A} \rightarrow \infty$, as $k \rightarrow \infty$.

Another problem of significant interest concerns the nonvanishing of Fourier coefficients of modular forms. A famous open problem is the conjecture on the Ramanujan function $\tau(n)$ due to Lehmer [14. Lehmer found that $\tau(n) \neq 0$ for $n \leq 10^{15}$ and conjectured that this is true for every integer $n$. Although this conjecture remains open, Serre [23, page 179] has made substantial progress by proving that $\tau(n)$ is nonzero for the vast majority of $n$.

The next result gives some complementary information, which is an analogue of [21, corollaire I], but the assumption of GRH is also removed.

Theorem 5. Let $A>0$, let $\eta \in\left(0, \frac{1}{31}\right)$ fixed, let $2 \mid k$ and let $\xi(k) \rightarrow \infty(k \rightarrow \infty)$ be a function satisfying $\xi(k) \leq \log _{3} k$. Then there is $f \in \mathrm{H}_{k, \mathrm{sym}^{2}}^{+}(1 ; \eta)$ such that, for $k \rightarrow \infty$,

$$
\sum_{\substack{p \leq(\log k)^{A} \\(p) \mid \leq\left(\xi(k) / \log _{3} k\right)^{1 / 2}}} \frac{1}{p}=\left(\log _{3} k\right)\left\{1+O_{A, \eta}\left(\frac{1}{\xi(k)}\right)\right\} .
$$

In what follows, $\eta \in\left(0, \frac{1}{2}\right)$ is a suitably fixed constant and we use $c_{1}, c_{2}, \ldots$ to denote positive constants depending on $\eta$ at most, which may take different values at each occurrence. Since all results of this paper are trivial when $k$ is bounded, we can suppose that $k \geq k_{0}(\eta)$, where $k_{0}(\eta)$ is a sufficiently large constant depending on $\eta$ such that both inequalities $(\log k)^{1000 / \eta} \leq k^{\eta}$ and $\log _{3} k \geq 1000$ hold for $k \geq k_{0}(\eta)$.

\section{ARChimedeAn FACTORS}

In order to prove our density theorem, we need a large sieve inequality. It is then necessary to investigate the corresponding Rankin-Selberg $L$-function. Since 
we are interested in the $k$-aspect, we have to explicitly evaluate the associated archimedean local factor, which is one of the main difficulties. Thanks to the recent work of Cogdell \& Michel [1] and the explanation of Cogdell (in private communication), we can compute the factor along the same line-via the local Langlands correspondence.

For $m \in \mathbb{N}, f \in \mathrm{H}_{k}^{*}(1)$ and $g \in \mathrm{H}_{k}^{*}(1)$, the Rankin-Selberg $L$-function of $\operatorname{sym}^{m} f$ and sym $^{m} g$ is given by

$$
L\left(s, \operatorname{sym}^{m} f \times \operatorname{sym}^{m} g\right):=\prod_{p} \prod_{0 \leq i, j \leq m}\left(1-\alpha_{f}(p)^{m-2 i} \alpha_{g}(p)^{m-2 j} p^{-s}\right)^{-1},
$$

where $\alpha_{f}(p)$ and $\alpha_{g}(p)$ are the "local roots" of $L_{p}\left(s, \operatorname{sym}^{m} f\right)$ and $L_{p}\left(s, \operatorname{sym}^{m} g\right)$, determined by (1.2) and (1.3).

The next result provides all the information we need.

Proposition 2.1. Let $2 \mid k$, let $f \in \mathrm{H}_{k}^{*}(1)$ and let $g \in \mathrm{H}_{k}^{*}(1)$.

(i) For $m \in \mathbb{N}$, the archimedean local factor of $L\left(s, \operatorname{sym}^{m} f \times \operatorname{sym}^{m} g\right)$ is

$$
\begin{aligned}
& L_{\infty}\left(s, \operatorname{sym}^{m} f \times \operatorname{sym}^{m} g\right) \\
& \quad=\Gamma_{\mathbb{R}}(s)^{\delta_{2 \mid m}} \Gamma_{\mathbb{C}}(s)^{[m / 2]+\delta_{2 \nmid m}} \prod_{\nu=1}^{m} \Gamma_{\mathbb{C}}(s+\nu(k-1))^{m-\nu+1},
\end{aligned}
$$

where $\Gamma_{\mathbb{R}}(s):=\pi^{-s / 2} \Gamma(s / 2), \Gamma_{\mathbb{C}}(s):=2(2 \pi)^{-s} \Gamma(s)$ and $\delta_{2 \mid n}:=1-\delta_{2 \nmid n}$.

(ii) Let $m=1,2,3,4$. Then the function

$$
\Lambda\left(s, \operatorname{sym}^{m} f \times \operatorname{sym}^{m} g\right):=L_{\infty}\left(s, \operatorname{sym}^{m} f \times \operatorname{sym}^{m} g\right) L\left(s, \operatorname{sym}^{m} f \times \operatorname{sym}^{m} g\right)
$$

is entire except possibly for simple poles at $s=0,1$ and satisfies the functional equation

$$
\Lambda\left(s, \operatorname{sym}^{m} f \times \operatorname{sym}^{m} g\right)=\varepsilon_{\text {sym }^{m}} f \times \operatorname{sym}^{m} g \Lambda\left(1-s, \operatorname{sym}^{m} f \times \operatorname{sym}^{m} g\right)
$$

with

$$
\varepsilon_{\mathrm{sym}^{m} f \times \operatorname{sym}^{m} g}= \pm 1 .
$$

Proof. Part (ii) comes from RS 2 and RS 3 of [22], and part (i) with $m=1$ is well known (see [7, Theorem 13.8]). Thus we only prove the assertion (i) for $m \geq 2$, which is done by the method in [1].

Following the notation in [1, we let $\ell \geq 2$ be an integer and let $D_{\ell}$ be the discrete series representation of $G L_{2}(\mathbb{R})$ of weight $\ell$. The representation $D_{\ell}$ corresponds to the infinite component of the automorphic representation associated to a classical cusp form of weight $\ell$. Let $W_{\mathbb{R}}$ be the Weil group of $\mathbb{R}$, which can be realized as $W_{\mathbb{R}}=\mathbb{C}^{\times} \cup j \mathbb{C}^{\times}$with $j^{2}=-1 \in \mathbb{C}^{\times}$and $j z j^{-1}=\bar{z}$ for $z \in \mathbb{C}^{\times}$. Then we introduce the following Weil group representations.

Let $\rho_{0}^{ \pm}$be the one-dimensional representations of $W_{\mathbb{R}}$ defined by $\rho_{0}^{ \pm}(z)=1$, $\rho_{0}^{+}(j)=1$ and $\rho_{0}^{-}(j)=-1$. Let $\mu \in \mathbb{C}$ such that $2 \mu=\ell-1 \in \mathbb{Z}$. Define the two-dimensional representation $\rho_{\ell}$ of $W_{\mathbb{R}}$ on the two-dimensional vector space $V_{2}=\left\langle e_{0}, e_{1}\right\rangle$ given by

$$
\begin{array}{ll}
\rho_{\ell}(z) e_{0}=(z / \bar{z})^{\mu} e_{0}, & \rho_{\ell}(z) e_{1}=(\bar{z} / z)^{\mu} e_{1}, \\
\rho_{\ell}(j) e_{0}=e_{1}, & \rho_{\ell}(j) e_{1}=(-1)^{\ell-1} e_{0} .
\end{array}
$$


In matrix form, for $z=r e^{i \theta}$, we can write

$$
\rho_{\ell}(z)=\left(\begin{array}{cc}
e^{i(\ell-1) \theta} & \\
& e^{-i(\ell-1) \theta}
\end{array}\right), \quad \rho_{\ell}(j)=\left(\begin{array}{ll} 
& (-1)^{\ell-1} \\
1 &
\end{array}\right) .
$$

Then under the local Langlands correspondence $\rho_{\ell}$ corresponds to $D_{\ell}$.

Now we compute $\operatorname{sym}^{m}\left(\rho_{k}\right) \otimes \operatorname{sym}^{m}\left(\rho_{k}\right)$. From Proposition 3.1 in [1], we have

$$
\operatorname{sym}^{m}\left(\rho_{k}\right)= \begin{cases}\bigoplus_{\nu=0}^{n} \rho_{(2 \nu+1)(k-1)+1} & \text { if } m=2 n+1, \\ \rho_{0}^{ \pm} \oplus \bigoplus_{\nu=1}^{n} \rho_{2 \nu(k-1)+1} & \text { if } m=2 n\end{cases}
$$

where $\rho_{0}^{+}$or $\rho_{0}^{-}$is selected when $n$ is even or odd, respectively.

We first consider the case $m=2 n+1$. By using (2.2), we have

$$
\begin{aligned}
\operatorname{sym}^{m}\left(\rho_{k}\right) \otimes \operatorname{sym}^{m}\left(\rho_{k}\right) & =\bigoplus_{\nu_{1}=0}^{n} \rho_{\left(2 \nu_{1}+1\right)(k-1)+1} \otimes \bigoplus_{\nu_{2}=0}^{n} \rho_{\left(2 \nu_{2}+1\right)(k-1)+1} \\
& =\bigoplus_{\nu_{1}=0}^{n} \bigoplus_{\nu_{2}=0}^{n} \rho_{\left(2 \nu_{1}+1\right)(k-1)+1} \otimes \rho_{\left(2 \nu_{2}+1\right)(k-1)+1} .
\end{aligned}
$$

It reduces to calculate $\rho_{a} \otimes \rho_{b}$ where $a, b \geq 2$. Tensoring the corresponding matrices, it follows that

$$
\rho_{a} \otimes \rho_{b}\left(r e^{i \theta}\right)=\left(\begin{array}{llll}
e^{i(a+b-1-1) \theta} & & & \\
& e^{i(a-b) \theta} & & \\
& & e^{-i(a-b) \theta} & \\
& & & e^{-i(a+b-1-1) \theta}
\end{array}\right)
$$

and

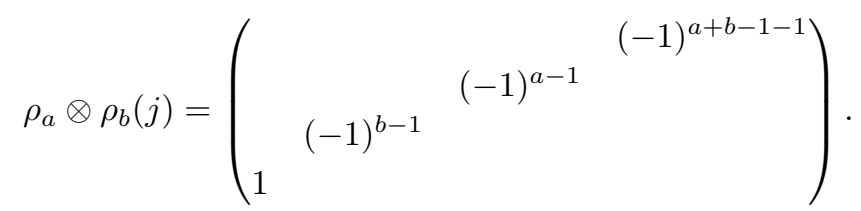

Thus $\rho_{a} \otimes \rho_{b}$ is decomposable with

$$
\left.\rho_{a} \otimes \rho_{b}\right|_{\left\langle e_{0} \otimes e_{0}, e_{1} \otimes e_{1}\right\rangle} \cong \rho_{a+b-1} .
$$

Moreover, for $a>b$, we see that $\left.\rho_{a} \otimes \rho_{b}\right|_{\left\langle e_{0} \otimes e_{1}, e_{1} \otimes e_{0}\right\rangle} \cong \rho_{a-b+1}$ via the change of basis

$$
e_{0} \otimes e_{1} \mapsto e_{0} \otimes e_{1}, \quad e_{1} \otimes e_{0} \mapsto(-1)^{b-1} e_{1} \otimes e_{0} .
$$

Reversing the roles of $a$ and $b$, we obtain $\left.\rho_{a} \otimes \rho_{b}\right|_{\left\langle e_{0} \otimes e_{1}, e_{1} \otimes e_{0}\right\rangle} \cong \rho_{b-a+1}$ for $a<b$. In the case $a=b$, it is reducible and indeed, $\left.\rho_{a} \otimes \rho_{a}\right|_{\left\langle e_{0} \otimes e_{1}, e_{1} \otimes e_{0}\right\rangle} \cong \rho_{0}^{+} \oplus \rho_{0}^{-}=: \rho_{1}$, say. Therefore for any $a, b \geq 2$, we have

$$
\rho_{a} \otimes \rho_{b} \cong \rho_{a+b-1} \oplus \rho_{|a-b|+1} .
$$


This immediately yields that

$$
\operatorname{sym}^{m}\left(\rho_{k}\right) \otimes \operatorname{sym}^{m}\left(\rho_{k}\right)=\bigoplus_{\nu_{1}=0}^{n} \bigoplus_{\nu_{2}=0}^{n} \rho_{2\left(\nu_{1}+\nu_{2}+1\right)(k-1)+1} \oplus \rho_{2\left|\nu_{1}-\nu_{2}\right|(k-1)+1} .
$$

From [12, we know that for $\ell \geq 2$,

$$
L\left(s, \rho_{\ell}\right)=\Gamma_{\mathbb{C}}\left(s+\frac{1}{2}(\ell-1)\right), \quad L\left(s, \rho_{0}^{+}\right)=\Gamma_{\mathbb{R}}(s), \quad L\left(s, \rho_{0}^{-}\right)=\Gamma_{\mathbb{R}}(s+1) .
$$

In view of the definition of $\rho_{1}$ and $\Gamma_{\mathbb{R}}(s) \Gamma_{\mathbb{R}}(s+1)=\Gamma_{\mathbb{C}}(s)$, the first relation in (2.3) also holds for $\ell=1$ from the last two. Thus we obtain

$$
\begin{aligned}
& L_{\infty}\left(s, \operatorname{sym}^{m} f \times \operatorname{sym}^{m} g\right) \\
& \quad=\prod_{\nu_{1}=0}^{n} \prod_{\nu_{2}=0}^{n} \Gamma_{\mathbb{C}}\left(s+\left(\nu_{1}+\nu_{2}+1\right)(k-1)\right) \Gamma_{\mathbb{C}}\left(s+\left|\nu_{1}-\nu_{2}\right|(k-1)\right),
\end{aligned}
$$

which is equivalent to the required formula, in view of

$$
\sum_{\substack{0 \leq \nu_{1}, \nu_{2} \leq n \\ \nu_{1}+\nu_{2}+1=\nu}} 1= \begin{cases}\nu & \text { if } 1 \leq \nu \leq n \\ m-\nu+1 & \text { if } n<\nu \leq m\end{cases}
$$

and

$$
\sum_{\substack{0 \leq \nu_{1}, \nu_{2} \leq n \\\left|\nu_{1}-\nu_{2}\right|=\nu}} 1= \begin{cases}n+1 & \text { if } \nu=0 \\ m-2 \nu+1 & \text { if } 1 \leq \nu \leq n\end{cases}
$$

For the case $m=2 n$, a similar argument yields the following formula:

$$
\begin{aligned}
\operatorname{sym}^{m}\left(\rho_{k}\right) \otimes \operatorname{sym}^{m}\left(\rho_{k}\right)=\rho_{0}^{+} & \oplus \bigoplus_{\nu=1}^{n} \rho_{2 \nu(k-1)+1} \oplus \bigoplus_{\nu=1}^{n} \rho_{2 \nu(k-1)+1} \\
& \oplus \bigoplus_{\nu_{1}=1}^{n} \bigoplus_{\nu_{2}=1}^{n} \rho_{2\left(\nu_{1}+\nu_{2}\right)(k-1)+1} \oplus \rho_{2\left|\nu_{1}-\nu_{2}\right|(k-1)+1 .} .
\end{aligned}
$$

From this and (2.3), we can obtain, as before, the desired result.

\section{BOUNDS FOR SYMMETRIC POWER $L$-FUNCTIONS}

In this section, we shall establish some estimates for the symmetric power $L$ functions in the weight aspect, which will be needed later. Since they are known or easy to prove, we shall briefly sketch the proof. We begin with the convexity bounds for $L\left(s, \operatorname{sym}^{m} f\right)$ and $L\left(s, \operatorname{sym}^{m} f \times \operatorname{sym}^{m} g\right)$ on the $k$-aspect.

Proposition 3.1. Let $m=1,2,3,4,2 \mid k$, let $f \in \mathrm{H}_{k}^{*}(1)$ and let $g \in \mathrm{H}_{k}^{*}(1)$. For any $\varepsilon>0$, we have

$$
L\left(s, \operatorname{sym}^{m} f\right) \ll_{\varepsilon} \begin{cases}(k+|\tau|)^{([m / 2]+1)(1-\sigma)+\varepsilon} & \text { if } 2 \nmid m, \\ (1+|\tau|)^{(1-\sigma) / 2}(k+|\tau|)^{[m / 2](1-\sigma)+\varepsilon} & \text { if } 2 \mid m\end{cases}
$$

and

$$
L\left(s, \operatorname{sym}^{m} f \times \operatorname{sym}^{m} g\right) \ll_{\varepsilon}(1+|\tau|)^{A_{m}(1-\sigma)}(k+|\tau|)^{B_{m}(1-\sigma)+\varepsilon}
$$

uniformly for $2 \mid k, 0 \leq \sigma \leq 1$ and $\tau \in \mathbb{R}$, where $[t]$ is the integral part of $t$, $A_{m}=(m+1) / 2$ and $B_{m}=m(m+1) / 2$.

We need an estimate for $\Gamma(s)$ in order to prove Proposition 3.1 . 
Lemma 3.1. Let $A>0$ be a fixed number and let $k_{0}=k_{0}(A)$ be a sufficiently large constant. Then for all sufficiently large $k \geq k_{0}$, we have

$$
\frac{\Gamma(k-s)}{\Gamma(k+s)} \ll_{A}(k+|\tau|)^{-2 \sigma}
$$

uniformly for $|\sigma| \leq A$, where the implied constants depend on $A$ only.

Proof. As usual we define the function $\log$ on $\mathbb{C} \backslash(-\infty, 0]$, the argument of which varies from $-\pi$ to $\pi$ anticlockwise. We apply Stirling's formula (see [26, § 4.42])

$$
\log \Gamma(s)=\left(s-\frac{1}{2}\right) \log s-s+\frac{\log (2 \pi)}{2}+\int_{0}^{\infty} \frac{\phi(u)}{(s+u)^{2}} \mathrm{~d} u,
$$

where $\phi(u):=-\int_{0}^{u}\left(\{v\}-\frac{1}{2}\right) \mathrm{d} v(\{v\}$ is the fractional part of $v)$. It is easy to show that

$$
|\phi(u)| \leq 1 \quad \text { and } \quad\left|\int_{0}^{\infty} \frac{\phi(u)}{(s+u)^{2}} \mathrm{~d} u\right| \leq 1 \quad(\sigma \geq 1) .
$$

The relations (3.3) and (3.4) allow us to deduce that for $|\sigma| \leq A$ and $k \geq k_{0}$,

$$
\begin{aligned}
-\Re e\left(\log \frac{\Gamma(k-s)}{\Gamma(k+s)}\right)= & \sigma \log \left((k-\sigma)^{2}+\tau^{2}\right)+\tau \arctan \left(\frac{2 \sigma \tau}{k^{2}-\sigma^{2}+\tau^{2}}\right) \\
& +\frac{1}{2}\left(k+\sigma-\frac{1}{2}\right) \log \left(\frac{(k+\sigma)^{2}+\tau^{2}}{(k-\sigma)^{2}+\tau^{2}}\right)+O_{A}(1) \\
= & \sigma \log \left((k-\sigma)^{2}+\tau^{2}\right)+O_{A}(1),
\end{aligned}
$$

which implies the required inequality.

We are now ready to prove Proposition 3.1, By (1.6), we have

$$
L\left(s, \operatorname{sym}^{m} f\right) \ll \zeta(1+\varepsilon)^{m+1} \ll_{\varepsilon} 1 \quad(s=1+\varepsilon+i \tau) .
$$

On the other hand, in view of the relation $\Gamma(s+1)=s \Gamma(s)$ and (1.7), we have

$$
\frac{L_{\infty}\left(1-s, \operatorname{sym}^{m} f\right)}{L_{\infty}\left(s, \operatorname{sym}^{m} f\right)}=(2 \pi)^{(n+1)(2 s-1)} \prod_{\nu=0}^{n}\left(\left(\nu+\frac{1}{2}\right)(k-1)-s\right) \frac{\Gamma\left(\left(\nu+\frac{1}{2}\right)(k-1)-s\right)}{\Gamma\left(\left(\nu+\frac{1}{2}\right)(k-1)+s\right)}
$$

if $m=2 n+1$, and

$$
\begin{aligned}
\frac{L_{\infty}\left(1-s, \operatorname{sym}^{m} f\right)}{L_{\infty}\left(s, \operatorname{sym}^{m} f\right)}= & \pi^{s-1 / 2}(2 \pi)^{n(2 s-1)} \frac{\Gamma\left(\frac{1+\delta_{2 \nmid n}-s}{2}\right)}{\Gamma\left(\frac{\delta_{2 \nmid n}+s}{2}\right)} \\
& \times \prod_{\nu=1}^{n}(\nu(k-1)-s) \frac{\Gamma(\nu(k-1)-s)}{\Gamma(\nu(k-1)+s)}
\end{aligned}
$$

if $m=2 n$.

From these, we use Stirling's formula or Lemma 3.1 (for $k$ small or large respectively) to deduce that for $s=-\varepsilon+i \tau$,

$$
\frac{L_{\infty}\left(1-s, \operatorname{sym}^{m} f\right)}{L_{\infty}\left(s, \operatorname{sym}^{m} f\right)} \ll_{\varepsilon, m} \begin{cases}(k+|\tau|)^{[m / 2]+1+\varepsilon} & \text { if } 2 \nmid m, \\ (1+|\tau|)^{1 / 2}(k+|\tau|)^{[m / 2]+\varepsilon} & \text { if } 2 \mid m .\end{cases}
$$

Thus (3.5) and the functional equation (1.9) imply that for $s=-\varepsilon+i \tau$,

$$
\left|L\left(s, \operatorname{sym}^{m} f\right)\right| \ll_{\varepsilon, m} \begin{cases}(k+|\tau|)^{[m / 2]+1+\varepsilon} & \text { if } 2 \nmid m, \\ (1+|\tau|)^{1 / 2}(k+|\tau|)^{[m / 2]+\varepsilon} & \text { if } 2 \mid m .\end{cases}
$$


Now the desired inequality (3.1) follows from (3.5) and (3.6) with the PhragménLindelöf theorem [26, §5.65]. The other one follows in the same way, with Proposition 2.1.

The next proposition contains the $k$-analogues of Lemmas 4.1 and 4.2 in [1]. We skip the proofs, as the methods are identical to the level cases.

Proposition 3.2. (i) Let $m=1,2,3,4$, let $2 \mid k$ and let $f \in \mathrm{H}_{k}^{*}(1)$. Then we have

$$
L\left(s, \operatorname{sym}^{m} f\right) \ll[\log (k|s|)]^{m+1}
$$

uniformly for $\sigma \geq 1-1 / \log (k|s|)$.

(ii) Let $m \in\{1,2,4\}$, let $2 \mid k$ and let $f \in \mathrm{H}_{k}^{*}(1)$. There is an absolute constant $c>0$ such that

uniformly for $\sigma=1$.

$$
L\left(s, \operatorname{sym}^{m} f\right) \gg[\log (k|s|)]^{-c}
$$

Remark 4 . The case $m=3$ in part (ii) is unknown due to the possibility of the exceptional zeros. (See [1] for a further discussion.)

Next we introduce the function $\log L\left(s, \mathrm{sym}^{m} f\right)$ and prepare some results for later use.

Let $m \in \mathbb{N}, 2 \mid k, f \in \mathrm{H}_{k}^{*}(1)$. Define

$$
\Lambda_{\mathrm{sym}^{m} f}(n)= \begin{cases}{\left[\alpha_{f}(p)^{m \nu}+\alpha_{f}(p)^{(m-2) \nu}+\cdots+\alpha_{f}(p)^{-m \nu}\right] \log p} & \text { if } n=p^{\nu} \\ 0 & \text { otherwise. }\end{cases}
$$

Then it is apparent that $\left|\Lambda_{\mathrm{sym}^{m} f}(n)\right| \leq(m+1) \log n(n \geq 1)$ and we have

$$
L(s):=-\frac{L^{\prime}}{L}\left(s, \operatorname{sym}^{m} f\right)=\sum_{n=1}^{\infty} \frac{\Lambda_{\mathrm{sym}^{m} f}(n)}{n^{s}}
$$

for $\sigma>1$. This follows easily from taking a logarithmic derivative on both sides of (1.4):

$$
\begin{aligned}
-\frac{L^{\prime}}{L}\left(s, \operatorname{sym}^{m} f\right) & =\sum_{p} \sum_{0 \leq j \leq m} \frac{\alpha_{f}(p)^{m-2 j} p^{-s} \log p}{1-\alpha_{f}(p)^{m-2 j} p^{-s}} \\
& =\sum_{p} \sum_{\nu \geq 1} \sum_{0 \leq j \leq m} \frac{\alpha_{f}(p)^{(m-2 j) \nu} \log p}{p^{s \nu}},
\end{aligned}
$$

which is equivalent to (3.8).

Suppose $f \in \mathrm{H}_{k, \operatorname{sym}^{m}}^{+}(1 ; \eta)$, where $\eta \in\left(0, \frac{1}{2}\right)$. Then $L(s)$ is holomorphic and zero-free in the region $\mathcal{S}$ (see (1.10) ), hence the integral of $L(s)$ from 2 to $s(s \in \mathcal{S})$ defines the $\operatorname{logarithm} \log L\left(s, \operatorname{sym}^{m} f\right)$, with the initial value taken as the usual natural logarithm of $L\left(2, \operatorname{sym}^{m} f\right)$. In particular, we have the absolutely convergent series

$$
\log L\left(s, \operatorname{sym}^{m} f\right)=\sum_{n=1}^{\infty} \frac{\Lambda_{\text {sym }^{m} f}(n)}{n^{s} \log n} \quad(\sigma>1)
$$

and the rather crude estimate

$$
\left|\log L\left(s, \operatorname{sym}^{m} f\right)\right| \leq(m+1) \zeta(\sigma) \ll_{m}(\sigma-1)^{-1} \quad(\sigma>1) .
$$


Let us write $\sigma_{0}=1-\eta$ for simplicity. The Borel-Carathedory theorem with the estimate (3.1) implies that for $\sigma>\sigma_{0}$ and $|\tau| \leq 100 k^{\eta}$,

$$
\log L\left(s, \operatorname{sym}^{m} f\right) \ll \frac{\log k}{\sigma-\sigma_{0}},
$$

where the implied constant is absolute. (See 4] for a detailed proof of the Dirichlet $L$-function case.) Similar to Lemma 7.1 of [21], we can easily prove a better estimate under GRH.

Proposition 3.3. Let $m=1,2,3,4$, let $2 \mid k$ and let $f \in \mathrm{H}_{k}^{*}(1)$. If $G R H$ for $L\left(s, \operatorname{sym}^{m} f\right)$ holds, then for any $\varepsilon>0$ and any $\alpha>\frac{1}{2}$ we have

$$
\log L\left(s, \operatorname{sym}^{m} f\right) \ll_{\varepsilon, \alpha}[\log (k|s|)]^{2(1-\sigma)+\varepsilon}
$$

uniformly for $\alpha \leq \sigma \leq 1$ and $\tau \in \mathbb{R}$.

Even without GRH, (3.11) can be refined for $f \in \mathrm{H}_{k, \mathrm{sym}^{m}}^{+}(1 ; \eta)$. To this end, we provide the $k$-analogue of Lemma 4.3 in [1].

Proposition 3.4. Let $\eta \in\left(0, \frac{1}{2}\right)$ fixed, let $\sigma_{0}=1-\eta$, let $m=1,2,3,4$, let $2 \mid k$, and let $f \in \mathrm{H}_{k, \mathrm{sym}^{m}}^{+}(1 ; \eta)$. Then we have

$$
\log L\left(s, \operatorname{sym}^{m} f\right)=\sum_{n=2}^{\infty} \frac{\Lambda_{\text {sym }^{m} f}(n)}{n^{s} \log n} e^{-n / T}+R
$$

uniformly for $2 \mid k, 3 \leq T \leq k^{\eta}, \sigma_{0}<\sigma \leq \frac{3}{2}$ and $|\tau| \leq T$, where

$$
R \ll_{\eta} T^{-\left(\sigma-\sigma_{0}\right) / 2}(\log k) /\left(\sigma-\sigma_{0}\right)^{2} .
$$

Further for any $0<\varepsilon<\frac{1}{4}$ and $\frac{1}{2}<\alpha<1$, under GRH for $L\left(s, \operatorname{sym}^{m} f\right)$ where $f \in \mathrm{H}_{k}^{*}(1)$, the asymptotic formula (3.13) holds uniformly for $\alpha \leq \sigma \leq \frac{3}{2}$ and $T \geq 1$, with

$$
R \ll_{\varepsilon, \alpha} T^{-(\sigma-\alpha)}(\log k)^{2(1-\alpha)+\varepsilon} .
$$

Proof. From the absolute convergence of (3.9) and the lemma in [27, § 7.9], we have

$$
\sum_{n=2}^{\infty} \frac{\Lambda_{\mathrm{sym}^{m} f}(n)}{n^{s} \log n} e^{-n / T}=\frac{1}{2 \pi i} \int_{2-i \infty}^{2+i \infty} \Gamma(z-s) \log L\left(z, \operatorname{sym}^{m} f\right) T^{z-s} \mathrm{~d} z .
$$

Now we deplace the line of integration $\Re e z=\kappa$ to the path $\mathcal{C}$ consisting of straight lines joining

$$
\kappa-i \infty, \quad \kappa-i 2 T, \quad \sigma_{1}-i 2 T, \quad \sigma_{1}+i 2 T, \quad \kappa+i 2 T, \quad \kappa+i \infty,
$$

where $\kappa:=1+1 / \log T$ and $\sigma_{1}:=\left(\sigma+\sigma_{0}\right) / 2$. By the residue theorem, it follows that

$$
\begin{aligned}
\sum_{n=2}^{\infty} \frac{\Lambda_{\mathrm{sym}^{m} f}(n)}{n^{s} \log n} e^{-n / T}= & \log L\left(s, \operatorname{sym}^{m} f\right) \\
& +\frac{1}{2 \pi i} \int_{\mathcal{C}} \Gamma(z-s) \log L\left(z, \operatorname{sym}^{m} f\right) T^{z-s} \mathrm{~d} z
\end{aligned}
$$


Then we estimate the contribution from each line segment of $\mathcal{C}$ in the last integral. Applying (3.10) and (3.11), we infer that the integral over $\mathcal{C}$ is

$$
\begin{aligned}
\ll & \frac{T^{\sigma_{1}-\sigma} \log k}{\sigma-\sigma_{0}} \int_{|y| \leq 3 T}\left|\Gamma\left(\sigma_{1}-\sigma+i y\right)\right| \mathrm{d} y \\
& +\frac{\log k}{\sigma-\sigma_{0}} \int_{\sigma_{1}}^{\kappa} T^{x-\sigma}|\Gamma(x-\sigma+i(T-\tau))| \mathrm{d} x \\
& +T^{1-\sigma} \int_{|y| \geq T}|\Gamma(\kappa-\sigma+i y)| \mathrm{d} y .
\end{aligned}
$$

To handle the gamma function, we use Stirling's formula of the form: for any fixed constant $c_{i}>0(i=1,2,3)$,

$$
|\Gamma(\sigma+i \tau)|=\sqrt{2 \pi}|\tau|^{\sigma-1 / 2} e^{-\pi|\tau| / 2}\left\{1+O\left(|\tau|^{-1}\right)\right\}
$$

for $-c_{1} \leq \sigma \leq c_{2}$ and $|\tau| \geq c_{3}$. Together with $|\Gamma(w)| \ll|w|^{-1}$ when $-\frac{1}{2} \leq \Re e w \leq c_{2}$ and $|\Im m w| \leq c_{3}$, the formula (3.13) with (3.14) follows plainly.

Under GRH, we can shift the line of integration in (3.16) to $\Re e z=\alpha^{\prime}:=\alpha-\varepsilon^{\prime}>$ $\frac{1}{2}$ where $\varepsilon^{\prime}:=\frac{1}{2} \min \left(\varepsilon, \alpha-\frac{1}{2}\right)>0$. Repeating the same argument, the remainder term in this case is

$$
\begin{aligned}
R & :=\frac{1}{2 \pi i} \int_{\alpha^{\prime}-i \infty}^{\alpha^{\prime}+i \infty} \Gamma(z-s) \log L\left(z, \operatorname{sym}^{m} f\right) T^{z-s} \mathrm{~d} z \\
& \ll_{\varepsilon, \alpha} T^{\alpha^{\prime}-\sigma}(\log k)^{2\left(1-\alpha^{\prime}\right)+\varepsilon} \int_{-\infty}^{\infty}\left|\Gamma\left(\alpha^{\prime}-\sigma+i y\right)\right|[\log (|y|+3)]^{2\left(1-\alpha^{\prime}\right)+\varepsilon} \mathrm{d} y \\
& \ll_{\varepsilon, \alpha} T^{-(\sigma-\alpha)-\varepsilon^{\prime}}(\log k)^{2(1-\alpha)+2 \varepsilon} \\
& \ll_{\varepsilon, \alpha} T^{-(\sigma-\alpha)}(\log k)^{2(1-\alpha)+2 \varepsilon}
\end{aligned}
$$

by (3.12) and $\alpha^{\prime}-\sigma \leq-\varepsilon^{\prime}$. This ends the proof after replacing $2 \varepsilon$ by $\varepsilon$.

Proposition 3.5. Let $\eta \in\left(0, \frac{1}{2}\right)$ fixed, let $m=1,2,3,4$ and let $2 \mid k$. Then for any $f \in \mathrm{H}_{k, \mathrm{sym}^{m}}^{+}(1 ; \eta)$, we have

$$
\log L\left(s, \operatorname{sym}^{m} f\right) \ll_{\eta} \frac{(\log k)^{4 \alpha / \eta}-1}{\alpha \log _{2} k}+\log _{3}(8 k)
$$

uniformly for $\sigma \geq 1-\alpha>1-\frac{1}{2} \eta$ and $|\tau| \leq(\log k)^{4 / \eta}$.

Proof. It suffices to consider $k \geq 16$ and $1-\alpha \leq \sigma \leq \frac{3}{2}$ in view of (3.10) and (3.11). We take $T=(\log k)^{4 / \eta}$ in Proposition 3.4 (recall $\left.\sigma_{0}=1-\eta\right)$, therefore the error term $R$ in (3.14) is $O(1)$, because of $\sigma-\sigma_{0} \geq \frac{1}{2} \eta$. Clearly the sum in (3.13) is

$$
\ll \sum_{p} p^{-\sigma} e^{-p / T}+O(1),
$$

so we may assume $\frac{1}{2} \leq \sigma \leq 1$. By Lemma 3.2 of [25], partial integration leads to the inequality

$$
\sum_{p \leq y} \frac{1}{p^{\sigma}} \ll \frac{y^{1-\sigma}-1}{(1-\sigma) \log y}+\log _{2} y
$$


uniformly for $\frac{1}{2} \leq \sigma \leq 1$ and $y \geq 3$. Hence we have

$$
\begin{aligned}
\sum_{p \leq T} p^{-\sigma} e^{-p / T} & \leq \sum_{p \leq T} p^{-\sigma} \\
& \ll \frac{(\log k)^{4(1-\sigma) / \eta}-1}{(1-\sigma) \log _{2} k}+\log _{3} k \\
& \ll \frac{(\log k)^{4 \alpha / \eta}-1}{\alpha \log _{2} k}+\log _{3} k .
\end{aligned}
$$

The contribution of $p>T$ can be estimated as

$$
\begin{aligned}
\sum_{p>T} p^{-\sigma} e^{-p / T} & \ll \int_{T}^{\infty} \frac{t^{-\sigma} e^{-t / T}}{\log t} \mathrm{~d} t \\
& =T^{1-\sigma} \int_{1}^{\infty} \frac{u^{-\sigma} e^{-u}}{\log (T u)} \mathrm{d} u \\
& \ll \frac{T^{1-\sigma}}{\log T} \ll \frac{(\log k)^{4(1-\sigma) / \eta}-1}{(1-\sigma) \log _{2} k} .
\end{aligned}
$$

Our assertion follows from inserting (3.21) and (3.22) into (3.19).

\section{A LARGe SIEVE INEQUALity}

Proposition 4.1. Let $m=1,2,3,4$, let $D_{m}:=m(m+1) / 4+1$, let $L \geq 1$ and let $\left\{a_{\ell}\right\}_{\ell \leq L}$ be a sequence of complex numbers. Then for any $\varepsilon>0$, we have

$$
\sum_{f \in \mathrm{H}_{k}^{*}(1)}\left|\sum_{\ell \leq L} a_{\ell} \lambda_{\mathrm{sym}^{m} f}(\ell)\right|^{2} \ll_{\varepsilon} k^{\varepsilon}\left(L+k^{D_{m}} L^{1 / 2+\varepsilon}\right) \sum_{\ell \leq L}\left|a_{\ell}\right|^{2} .
$$

Proof. By the duality principle (which follows from the same norms of a Hilbert space operator and its adjoint), it suffices to show

$$
\sum_{\ell \leq L}\left|\sum_{f \in \mathrm{H}_{k}^{*}(1)} b_{f} \lambda_{\mathrm{sym}^{m} f}(\ell)\right|^{2} \ll_{\varepsilon} k^{\varepsilon}\left(L+k^{D_{m}} L^{1 / 2+\varepsilon}\right) \sum_{f \in \mathrm{H}_{k}^{*}(1)}\left|b_{f}\right|^{2}
$$

for any sequence of complex numbers $\left\{b_{f}\right\}_{f \in \mathrm{H}_{k}^{*}(1)}$.

The left-hand side of (4.1) is

$$
\begin{aligned}
& \ll \sum_{\ell \geq 1}\left|\sum_{f \in \mathrm{H}_{k}^{*}(1)} b_{f} \lambda_{\mathrm{sym}^{m} f}(\ell)\right|^{2} e^{-\ell / L} \\
& =\sum_{f, g \in \mathrm{H}_{k}^{*}(1)} b_{f} \overline{b_{g}} \sum_{\ell \geq 1} \lambda_{\mathrm{sym}^{m} f}(\ell) \lambda_{\mathrm{sym}^{m} g}(\ell) e^{-\ell / L} .
\end{aligned}
$$

From the well-known formula

$$
\frac{1}{2 \pi i} \int_{(c)} \Gamma(w) y^{w} \mathrm{~d} w=e^{-1 / y} \quad(\text { for } c>0)
$$


we obtain, together with a shift of line of integration,

$$
\begin{aligned}
\sum_{\ell \geq 1} \lambda_{\operatorname{sym}^{m} f}(\ell) \lambda_{\operatorname{sym}^{m} g}(\ell) e^{-\ell / L} \\
=\frac{1}{2 \pi i} \int_{(2)} L\left(s, \operatorname{sym}^{m} f \times \operatorname{sym}^{m} g\right) \Gamma(s) L^{s} \mathrm{~d} s \\
=\operatorname{Res}_{s=1} L\left(s, \operatorname{sym}^{m} f \times \operatorname{sym}^{m} g\right) \Gamma(s) L^{s} \\
\quad+\frac{1}{2 \pi i} \int_{(1 / 2+\varepsilon)} L\left(s, \operatorname{sym}^{m} f \times \operatorname{sym}^{m} g\right) \Gamma(s) L^{s} \mathrm{~d} s .
\end{aligned}
$$

The residue term comes only for $f=g$ by [1, Section 5.1]. With the estimate (3.2), we have

$$
\begin{aligned}
\operatorname{Res}_{s=1} L\left(s, \operatorname{sym}^{m} f \times \operatorname{sym}^{m} g\right) \Gamma(s) L^{s} & =\lim _{s \rightarrow 1+}(s-1) L\left(s, \operatorname{sym}^{m} f \times \operatorname{sym}^{m} g\right) \Gamma(s) L^{s} \\
& \ll \varepsilon k^{\varepsilon} \delta_{f, g} L,
\end{aligned}
$$

where $\delta_{f, g}=1$ if $f=g$, and $=0$ otherwise.

The last integral in (4.4) is $\ll k^{m(m+1) / 4+\varepsilon} L^{1 / 2+\varepsilon}$, by (3.2) again and (3.17). Therefore,

$$
\sum_{\ell \geq 1} \lambda_{\operatorname{sym}^{m} f}(\ell) \lambda_{\text {sym }^{m} g}(\ell) e^{-\ell / L} \ll k^{\varepsilon}\left(\delta_{f, g} L+k^{m(m+1) / 4} L^{1 / 2+\varepsilon}\right) .
$$

Inserting (4.5) into (4.2) with $\mathrm{H}_{k}^{*}(1) \ll k$, we obtain the result.

\section{Proof of Theorem 1}

Our proof is based on the method of Montgomery in [16], but at first, we show a factorization to prepare a convenient mollifier for zero detection. The approach here was kindly suggested by the referee.

Lemma 5.1. Let $m \in \mathbb{N}, z>(m+1)^{2}$ be any fixed number and let $P(z)=\prod_{p \leq z} p$. For any $\sigma>1$, we have

$$
L\left(s, \operatorname{sym}^{m} f\right)^{-1}=G_{f}(s) \sum_{(n, P(z))=1} \lambda_{\operatorname{sym}^{m} f}(n) \mu(n) n^{-s},
$$

where the Dirichlet series $G_{f}(s)$ converges absolutely for $\sigma>\frac{1}{2}$, and $G_{f}(s) \ll_{m, z, \varepsilon} 1$ uniformly for $\sigma>\frac{1}{2}+\varepsilon$.

Proof. By (1.6), we have $\left|\lambda_{\text {sym }^{m} f}(p)\right| \leq m+1$ so $1-\lambda_{\text {sym }^{m} f}(p) p^{-s}$ is nonzero for $\sigma \geq \frac{1}{2}$ and $p>z$, from our choice of $z$. Formally, we can write

$$
\begin{aligned}
G_{f}(s):= & \prod_{p \leq z} \prod_{j=0}^{m}\left(1-\alpha_{f}(p)^{m-2 j} p^{-s}\right) \\
& \times \prod_{p>z}\left\{\left(1-\lambda_{\text {sym }^{m}} f(p) p^{-s}\right)^{-1} \prod_{j=0}^{m}\left(1-\alpha_{f}(p)^{m-2 j} p^{-s}\right)\right\},
\end{aligned}
$$

by (1.4). If $p>z$, the $p$-local factor of $G_{f}(s)$ is of the form $1+O_{m}\left(p^{-2 \sigma}\right)$, whence both the absolute and uniform convergence of $G_{f}(s)$ are justified in our specified regions. 
Remark 5. Such a factorization (together with other delicate methods) is applied in Kowalski \& Michel [13] to count the zeros of automorphic $L$-functions on $G L(n)$.

Now we are in a position to prove Theorem 1, Let us make two simple observations. First an argument similar to [27, §9.2] yields that

$$
N\left(\frac{1}{2}, j, \operatorname{sym}^{m} f\right)-N\left(\frac{1}{2}, j-1, \operatorname{sym}^{m} f\right) \ll \log (k j) .
$$

Thus the result of Theorem 1 is trivial if $T \geq k^{r}$, in view of (1.1).

Second the case $1 \leq T \leq(\log k)^{3}$ can be deduced from the particular case $T=(\log k)^{3}$, by (5.1) again. Therefore we assume

$$
(\log k)^{3} \leq T \leq k^{r} .
$$

We cut the rectangle $\alpha \leq \sigma \leq 1$ and $0 \leq \tau \leq T$ horizontally into boxes of width $2(\log k)^{2}$. By (5.1) each box $\alpha \leq \sigma \leq 1$ and $Y \leq \tau \leq Y+2(\log k)^{2}$ contains at most $O\left((\log k)^{3}\right)$ zeros. Let $n_{\mathrm{sym}^{m} f}$ be the number of boxes which contain at least one zero $\rho$ of $L\left(s, \operatorname{sym}^{m} f\right)$. Then

$$
N\left(\alpha, T, \operatorname{sym}^{m} f\right) \ll n_{\text {sym }^{m} f}(\log k)^{3} .
$$

We shall complete the proof by showing that

$$
\sum_{f \in \mathrm{H}_{k}^{*}(1)} n_{\mathrm{sym}^{m} f} \ll_{r, \varepsilon} T k^{E_{m, r}(1-\alpha) /(3-2 \alpha)+\varepsilon} .
$$

Consider $\alpha \geq \frac{1}{2}+2 \varepsilon$. Let $x, y \in\left[1, k^{10 m^{2}(1+r)}\right]$ and define

$$
M_{x}\left(s, \operatorname{sym}^{m} f\right)=G_{f}(s) \sum_{\substack{\ell \leq x \\(\ell, P(z))=1}} \mu(\ell) \lambda_{\mathrm{sym}^{m} f}(\ell) \ell^{-s},
$$

where $G_{f}(s)$ and $P(z)$ are defined as in Lemma 5.1. By Lemma 5.1, for $\sigma>\frac{1}{2}$ we have

$$
1=\left(1-L\left(s, \operatorname{sym}^{m} f\right) M_{x}\left(s, \operatorname{sym}^{m} f\right)\right)+L\left(s, \operatorname{sym}^{m} f\right) M_{x}\left(s, \operatorname{sym}^{m} f\right) .
$$

Let $\rho=\beta+i \gamma$ with $\beta \geq \alpha\left(>\frac{1}{2}+\varepsilon\right)$ and $0 \leq \gamma \leq T$ be a zero of $L\left(s, \operatorname{sym}^{m} f\right)$, and write

$$
\kappa=1 / \log k, \quad \kappa_{1}=1-\beta+\kappa(>0) \quad \text { and } \quad \kappa_{2}=\frac{1}{2}-\beta+\varepsilon(<0) .
$$

In view of the preceding identity and (4.3) with $c=\kappa_{1}$, we obtain

$$
\begin{array}{r}
e^{-1 / y}=\frac{1}{2 \pi i} \int_{\left(\kappa_{1}\right)}\left(1-L\left(\rho+w, \operatorname{sym}^{m} f\right) M_{x}\left(\rho+w, \operatorname{sym}^{m} f\right)\right) \Gamma(w) y^{w} \mathrm{~d} w \\
+\frac{1}{2 \pi i} \int_{\left(\kappa_{1}\right)} L\left(\rho+w, \operatorname{sym}^{m} f\right) M_{x}\left(\rho+w, \operatorname{sym}^{m} f\right) \Gamma(w) y^{w} \mathrm{~d} w .
\end{array}
$$

Observing that the zero of $L\left(\rho+w, \operatorname{sym}^{m} f\right)$ cancels the simple pole of $\Gamma(w)$ at $w=0$, we translate the line of integration of the second integral to $\Re e w=\kappa_{2}$ without introducing extra terms. Thus we have

$$
\begin{array}{r}
e^{-1 / y}=\frac{1}{2 \pi i} \int_{\left(\kappa_{1}\right)}\left(1-L\left(\rho+w, \operatorname{sym}^{m} f\right) M_{x}\left(\rho+w, \operatorname{sym}^{m} f\right)\right) \Gamma(w) y^{w} \mathrm{~d} w \\
+\frac{1}{2 \pi i} \int_{\left(\kappa_{2}\right)} L\left(\rho+w, \operatorname{sym}^{m} f\right) M_{x}\left(\rho+w, \operatorname{sym}^{m} f\right) \Gamma(w) y^{w} \mathrm{~d} w .
\end{array}
$$


Next we estimate the contribution of $|\Im m w| \geq(\log k)^{2}$ in the integrals of (5.4). Note that for $\Re e w=\kappa_{2}=\frac{1}{2}-\beta+\varepsilon$, we have the convexity bound (by (3.1))

$$
L\left(\rho+w, \operatorname{sym}^{m} f\right) \ll(k+T+|\Im m w|)^{(m+1) / 4+\varepsilon}
$$

and the trivial estimate (with (1.6) and $G_{f}(s) \ll_{\varepsilon} 1$ by Lemma 5.1)

$$
M_{x}\left(\rho+w, \operatorname{sym}^{m} f\right) \ll_{\varepsilon} x^{1 / 2+\varepsilon} .
$$

Thus the contribution of $|\Im m w| \geq(\log k)^{2}$ to the second integral of (5.4) is

$$
\begin{aligned}
& \ll_{\varepsilon} x^{1 / 2+\varepsilon} y^{1 / 2-\alpha} \int_{|\Im m w| \geq(\log k)^{2}}(k+T+|\Im m w|)^{(m+1) / 4+\varepsilon}|\Gamma(w)||\mathrm{d} w| \\
& \ll_{\varepsilon} x^{1 / 2+\varepsilon} y^{1 / 2-\alpha}(k+T)^{(m+1) / 4+\varepsilon} e^{-(\log k)^{2}} \\
& \ll_{\varepsilon, r} 1 / k
\end{aligned}
$$

by (5.5) and $T \leq k^{r}$.

By (1.5) and (1.6), $L\left(s, \operatorname{sym}^{m} f\right) \ll \zeta(\sigma)^{m+1}$ for $\sigma>1$. Together with Lemma 5.1, we get that for $\Re e w=\kappa_{1}=1-\beta+\kappa$ and $x \geq 1$,

$$
\begin{aligned}
1- & L\left(\rho+w, \operatorname{sym}^{m} f\right) M_{x}\left(\rho+w, \operatorname{sym}^{m} f\right) \\
& =L\left(\rho+w, \operatorname{sym}^{m} f\right) G_{f}(\rho+w) \sum_{\ell>x,(\ell, P(z))=1} \frac{\mu(\ell) \lambda_{\mathrm{sym}^{m} f}(\ell)}{\ell^{\rho+w}} \\
& \ll_{\varepsilon} \zeta(1+\kappa)^{m+1} \sum_{\ell \geq 1} \mathrm{~d}_{m+1}(\ell) \ell^{-(1+\kappa)} \\
& \ll_{\varepsilon} k^{\varepsilon} .
\end{aligned}
$$

Hence the portion of $|\Im m w| \geq(\log k)^{2}$ in the first integral of (5.4) is

$$
\ll_{\varepsilon} y^{1-\alpha} k^{\varepsilon} e^{-(\log k)^{2}} \ll_{\varepsilon} 1 / k .
$$

Inserting (5.7) and (5.9) into (5.4), the remnant of the right side in (5.4) is $\gg 1$. Noting the fact that $1 \leq C(a+b) \Rightarrow 1 \leq 2 C^{2}\left(a+b^{2}\right)$ (where $a>0, b>0, C \geq 1$ ), we deduce with Cauchy-Schwarz's inequality that

$$
\begin{aligned}
1 \ll & k^{\varepsilon} y^{2(1-\alpha)} \\
& \times \int_{-K}^{K}\left|1-L\left(1+\kappa+i(\gamma+v), \operatorname{sym}^{m} f\right) M_{x}\left(1+\kappa+i(\gamma+v), \operatorname{sym}^{m} f\right)\right|^{2} \mathrm{~d} v \\
& +y^{1 / 2-\alpha} \int_{-K}^{K}\left|L\left(\frac{1}{2}+\varepsilon+i(\gamma+v), \operatorname{sym}^{m} f\right) M_{x}\left(\frac{1}{2}+\varepsilon+i(\gamma+v), \operatorname{sym}^{m} f\right)\right| \mathrm{d} v,
\end{aligned}
$$

where $K:=(\log k)^{2}$.

We label the boxes and separate them into two groups, for the odd-indexed and the even-indexed, respectively. This ensures the separation between two zeros from distinct boxes in the same group of at least $2(\log k)^{2}$. Therefore, the number of 
boxes which contain at least a zero is

$$
\begin{aligned}
& n_{\text {sym }^{m} f} \\
& \ll k^{\varepsilon} y^{2(1-\alpha)} \int_{0}^{2 T}\left|1-L\left(1+\kappa+i v, \operatorname{sym}^{m} f\right) M_{x}\left(1+\kappa+i v, \operatorname{sym}^{m} f\right)\right|^{2} \mathrm{~d} v \\
& \quad+y^{1 / 2-\alpha} \int_{0}^{2 T}\left|L\left(\frac{1}{2}+\varepsilon+i v, \operatorname{sym}^{m} f\right) M_{x}\left(\frac{1}{2}+\varepsilon+i v, \operatorname{sym}^{m} f\right)\right| \mathrm{d} v \\
& =: k^{\varepsilon}\left(y^{2(1-\alpha)} I_{\text {sym }^{m} f}^{\prime}+y^{1 / 2-\alpha} I_{\text {sym }^{m} f}^{\prime \prime}\right), \quad \text { say. }
\end{aligned}
$$

Apparently (5.5) and (5.6) imply

$$
I_{\mathrm{sym}^{m} f}^{\prime \prime} \ll_{r, \varepsilon} T x^{1 / 2+\varepsilon} k^{r(m+1) / 4+r \varepsilon},
$$

for $T \leq k^{r}$. Similarly to (5.8), we can write

$$
\begin{aligned}
& 1-L\left(1+\kappa+i v, \operatorname{sym}^{m} f\right) M_{x}\left(1+\kappa+i v, \operatorname{sym}^{m} f\right) \\
& \ll_{\varepsilon, m} k^{\varepsilon}\left|\sum_{\substack{x<\ell \leq X \\
(\ell, P(z))=1}} \frac{\mu(\ell) \lambda_{\mathrm{sym}^{m} f}(\ell)}{\ell^{1+\kappa+i v}}\right|+k^{\varepsilon} \sum_{\ell>X} \frac{\mathrm{d}_{m+1}(\ell)}{\ell^{1+\kappa}},
\end{aligned}
$$

where $X=e^{4(\log k)^{2}}$. Splitting $\ell^{-\kappa / 2}$ out of the second sum, this term is

$$
\ll_{\varepsilon, m} k^{\varepsilon} X^{-\kappa / 2} \zeta(1+\kappa / 2)^{m+1} \ll k^{-1} .
$$

Thus

$$
\sum_{f \in \mathrm{H}_{k}^{*}(1)} I_{\mathrm{sym}^{m} f}^{\prime} \ll k^{\varepsilon} \int_{0}^{2 T} \sum_{f \in \mathrm{H}_{k}^{*}(1)}\left|\sum_{\substack{x<\ell \leq X \\(\ell, P(\bar{z}))=1}} \frac{\mu(\ell) \lambda_{\mathrm{sym}^{m} f}(\ell)}{\ell^{1+\kappa+i v}}\right|^{2} \mathrm{~d} v+T .
$$

For any $L \in[x, X]$, we apply Proposition 4.1 with the sequence $a_{\ell}=\mu(\ell) \ell^{-(1+\kappa+i v)}$ for $L<\ell \leq 2 L$ with $(\ell, P(z))=1$ and 0 otherwise to get

$$
\sum_{f \in \mathrm{H}_{k}^{*}(1)}\left|\sum_{\substack{L<\ell \leq 2 L \\(\ell, P(\bar{z}))=1}} \frac{\mu(\ell) \lambda_{\mathrm{sym}^{m} f}(\ell)}{\ell^{1+\kappa+i v}}\right|^{2} \ll k^{\varepsilon}\left(L+k^{D_{m}} L^{1 / 2+\varepsilon}\right) L^{-1-2 \kappa} .
$$

Separating the range $x<\ell \leq X$ in (5.12) into dyadic intervals, it follows with Cauchy-Schwarz's inequality that

$$
\sum_{f \in \mathrm{H}_{k}^{*}(1)} I_{\mathrm{sym}^{2} f}^{\prime} \ll_{r, \varepsilon} k^{2 \varepsilon} T\left(1+k^{D_{m}} x^{-1 / 2+\varepsilon}\right) .
$$

Thus we conclude from (5.10) and (5.11) that

$$
\sum_{f \in \mathrm{H}_{k}^{*}(1)} n_{\mathrm{Sym}^{m} f} \ll_{r, \varepsilon} T x^{\varepsilon} k^{2 r \varepsilon}\left\{y^{2(1-\alpha)}\left(1+k^{D_{m}} x^{-1 / 2}\right)+y^{1 / 2-\alpha} x^{1 / 2} k^{r(m+1) / 4}\right\} .
$$

Taking $x=k^{2 D_{m}}$ and $y=k^{E_{m, r} /(2(3-2 \alpha))}$, the proof of (5.3), hence Theorem 1, is complete with $\varepsilon /\left(2\left(D_{m}+r\right)\right)$ in place of $\varepsilon$. 


\section{Moments of $L\left(1, \operatorname{sym}^{m} f\right)$}

The aim of this section is to prove Proposition 6.1 below. We first introduce the preliminary notation: for $\theta \in \mathbb{R}, m \in \mathbb{N}$ and $|x|<1$,

$$
\begin{aligned}
g(\theta) & :=\operatorname{diag}\left[e^{i \theta}, e^{-i \theta}\right] \\
\operatorname{sym}^{m}[g(\theta)] & :=\operatorname{diag}\left[e^{i m \theta}, e^{i(m-2) \theta}, \ldots, e^{-i m \theta}\right], \\
\operatorname{tr}\left(\operatorname{sym}^{m}[g(\theta)]\right) & :=\sum_{0 \leq j \leq m} e^{i(m-2 j) \theta}=\sin [(m+1) \theta] / \sin \theta \\
D\left(x, \operatorname{sym}^{m}[g(\theta)]\right) & :=\operatorname{det}\left(I-x \cdot \operatorname{sym}^{m}[g(\theta)]\right)^{-1}=\prod_{0 \leq j \leq m}\left(1-e^{i(m-2 j) \theta} x\right)^{-1} .
\end{aligned}
$$

Let $z \in \mathbb{C}$. For $m \in \mathbb{N}$ and $\nu \geq 0$, we define $\lambda_{m}^{z, \nu}[g(\theta)]$ by

$$
D\left(x, \operatorname{sym}^{m}[g(\theta)]\right)^{z}=\sum_{\nu \geq 0} \lambda_{m}^{z, \nu}[g(\theta)] x^{\nu} \quad(|x|<1) .
$$

Following the definitions, we have

$$
\begin{aligned}
\lambda_{m}^{1,1}[g(\theta)] & =\operatorname{tr}\left(\operatorname{sym}^{m}[g(\theta)]\right)=\frac{\sin [(m+1) \theta]}{\sin \theta}, \\
\log D\left(x, \operatorname{sym}^{m}[g(\theta)]\right) & =\operatorname{tr}\left(\operatorname{sym}^{m}[g(\theta)]\right) x+O\left(x^{2}\right) \quad(|x|<1) .
\end{aligned}
$$

Besides, let $\theta_{f}(p) \in[0, \pi]$ such that $\alpha_{f}(p)=e^{i \theta_{f}(p)}$ which is admissible by (1.3). Then it is seen that by (1.2) and (1.3),

$$
\lambda_{f}\left(p^{m}\right)=\frac{\sin \left[(m+1) \theta_{f}(p)\right]}{\sin \theta_{f}(p)}=\operatorname{tr}\left(\operatorname{sym}^{m}\left[g\left(\theta_{f}(p)\right)\right]\right)=\lambda_{m}^{1,1}\left[g\left(\theta_{f}(p)\right)\right] .
$$

Moreover, it is evident that from (6.1) and (1.4),

$$
L\left(s, \operatorname{sym}^{m} f\right)^{z}=\prod_{p} D\left(p^{-s}, \operatorname{sym}^{m}\left[g\left(\theta_{f}(p)\right)\right]\right)^{z} \quad(\sigma>1),
$$

whence $L\left(s, \operatorname{sym}^{m} f\right)^{z}$ admits a Dirichlet series

$$
L\left(s, \operatorname{sym}^{m} f\right)^{z}=\sum_{n \geq 1} \lambda_{\mathrm{sym}^{m} f}^{z}(n) n^{-s} \quad(\sigma>1),
$$

where $\lambda_{\mathrm{sym}^{m} f}^{z}(n)$ is multiplicative and by (6.2),

$$
\lambda_{\text {sym }^{m} f}^{z}\left(p^{\nu}\right)=\lambda_{m}^{z, \nu}\left[g\left(\theta_{f}(p)\right)\right] .
$$

Remark 6 . The symbols in (6.1), though a bit heavy, carry interpretations in representation theory. The coefficients $\lambda_{m}^{z, \nu}[g(\theta)]$ can be viewed as a function generated by the characters of $\mathrm{SU}(2)$. The combinatorial structure of $\lambda_{\mathrm{sym}^{m} f}^{z}\left(p^{\nu}\right)$ is encrypted in the decomposition of $\lambda_{m}^{z, \nu}[g(\theta)]$ into irreducible characters. Furthermore, the Petersson formula (a main ingredient in our proof) embodies the interpretation as the equidistribution of a certain family of tuples of conjugacy classes. These are part of the salient points in [1, where readers will find the details.

In the sequel, we write

$$
\omega(f):=\frac{2 \pi^{2}}{(k-1) L\left(1, \operatorname{sym}^{2} f\right)}
$$


and

$$
M_{\mathrm{sym}^{m}}^{z}:=\prod_{p} \frac{2}{\pi} \int_{0}^{\pi} D\left(p^{-1}, \operatorname{sym}^{m}[g(\theta)]\right)^{z} \sin ^{2} \theta \mathrm{d} \theta .
$$

Proposition 6.1. Let $\eta \in\left(0, \frac{1}{31}\right)$ fixed, let $m=1,2,3,4$ and let $2 \mid k$. Then there are two positive constants $\delta=\delta(\eta)$ and $c=c(\eta)$ such that

$$
\sum_{f \in \mathrm{H}_{k, \mathrm{sym}}^{+} m(1 ; \eta)} \omega(f) L\left(1, \operatorname{sym}^{m} f\right)^{z}=M_{\mathrm{sym}^{m}}^{z}+O_{\eta}\left(e^{-\delta \log k / \log _{2} k}\right)
$$

uniformly for

$$
2 \mid k \quad \text { and } \quad|z| \leq c \log k / \log _{2}(8 k) \log _{3}(8 k) .
$$

The same asymptotic formula with $\mathrm{H}_{k}^{*}(1)$ in place of $\mathrm{H}_{k, \mathrm{sym}^{m}}^{+}(1 ; \eta)$ also holds if either $\Re e z \geq 0$ or $\Re e z<0$, but $m \neq 3$.

We need a couple of lemmas to prove Proposition 6.1. Our first lemma is to express $\lambda_{\text {sym }^{m} f}^{z}\left(p^{\nu}\right)$ in terms of $\lambda_{f}\left(p^{\nu^{\prime}}\right)$. Cogdell \& Michel [1] achieve it in a more general context of compact groups. But for the case $\mathrm{SU}(2)$, we choose to give a direct and "elementary" approach which is more straightforward to the reader not used to the language of representation theory.

Lemma 6.1. Let $2 \mid k$, let $m \in \mathbb{N}$, let $z \in \mathbb{C}$ and let $f \in \mathrm{H}_{k}^{*}(1)$. Then for any prime $p$ and integer $\nu \geq 0$, we have

$$
\lambda_{\mathrm{sym}^{m} f}^{z}\left(p^{\nu}\right)=\sum_{0 \leq \nu^{\prime} \leq m \nu} \mu_{m, \nu^{\prime}}^{z, \nu} \lambda_{f}\left(p^{\nu^{\prime}}\right),
$$

where

$$
\mu_{m, \nu^{\prime}}^{z, \nu}=\frac{2}{\pi} \int_{0}^{\pi} \lambda_{m}^{z, \nu}[g(\theta)] \sin \left[\left(\nu^{\prime}+1\right) \theta\right] \sin \theta \mathrm{d} \theta .
$$

Further, letting $\delta(a, b)$ be 1 for $a=b$ and 0 otherwise, we have

$$
\begin{aligned}
\mu_{m, \nu^{\prime}}^{z, 1} & =z \delta\left(m, \nu^{\prime}\right) \quad\left(0 \leq \nu^{\prime} \leq m\right), \\
\left|\mu_{m, \nu^{\prime}}^{z, \nu}\right| & \leq\left(\begin{array}{c}
(m+1)|z|+\nu-1 \\
\nu
\end{array}\right) \quad\left(0 \leq \nu^{\prime} \leq m \nu\right) .
\end{aligned}
$$

Proof. We start with the observation that $\theta \mapsto D\left(x, \operatorname{sym}^{m}[g(\theta)]\right)^{z}$ is even and

$$
\lambda_{m}^{z, \nu}[g(\theta)]=\left.\frac{1}{\nu !} \frac{\mathrm{d}^{\nu}}{\mathrm{d} x^{\nu}} D\left(x, \operatorname{sym}^{m}[g(\theta)]\right)^{z}\right|_{x=0} .
$$

It follows that the function $\theta \mapsto \lambda_{m}^{z, \nu}[g(\theta)]$ is also even. Hence $\lambda_{m}^{z, \nu}[g(\theta)] \sin \theta$ is an odd function and a polynomial in $e^{i \theta}$ of degree $\leq m \nu+1$. It is plain that $\mu_{m, \nu^{\prime}}^{z, \nu}$ defined in (6.10) is the coefficient of the Fourier (sine) series

$$
\lambda_{m}^{z, \nu}[g(\theta)] \sin \theta=\sum_{0 \leq \nu^{\prime} \leq m \nu} \mu_{m, \nu^{\prime}}^{z, \nu} \sin \left[\left(\nu^{\prime}+1\right) \theta\right] .
$$

We thus obtain (6.9) in view of (6.8) and (6.5).

By the series expansion

$$
(1-x)^{-z}=\sum_{\nu \geq 0}\left(\begin{array}{c}
z+\nu-1 \\
\nu
\end{array}\right) x^{\nu}
$$


valid for $|x|<1$ and $z \in \mathbb{C}$, where

$$
\left(\begin{array}{l}
z \\
\nu
\end{array}\right):=\frac{1}{\nu !} \prod_{j=0}^{\nu-1}(z-j)
$$

with the convention $\left(\begin{array}{l}z \\ 0\end{array}\right)=1$, we have from the definition in (6.1) that

$$
\begin{aligned}
D\left(x, \operatorname{sym}^{m}[g(\theta)]\right)^{z} & =\prod_{0 \leq j \leq m} \sum_{\nu_{j} \geq 0}\left(\begin{array}{c}
z+\nu_{j}-1 \\
\nu_{j}
\end{array}\right)\left(e^{i(m-2 j) \theta} x\right)^{\nu_{j}} \\
& =\sum_{\nu \geq 0} x^{\nu} \sum_{\substack{\vec{\nu} \in \mathbb{N}^{m+1} \\
|\vec{\nu}|=\nu}} \prod_{0 \leq j \leq m}\left(\begin{array}{c}
z+\nu_{j}-1 \\
\nu_{j}
\end{array}\right) e^{i(m-2 j) \nu_{j} \theta},
\end{aligned}
$$

where $\vec{\nu}:=\left(\nu_{0}, \ldots, \nu_{m}\right)$ and $|\vec{\nu}|:=\nu_{0}+\cdots+\nu_{m}$. Thus by comparing with (6.2), we get

$$
\lambda_{m}^{z, \nu}[g(\theta)]=\sum_{\substack{\vec{\nu} \in \mathbb{N}^{m+1} \\
|\vec{\nu}|=\nu}} \prod_{0 \leq j \leq m}\left(\begin{array}{c}
z+\nu_{j}-1 \\
\nu_{j}
\end{array}\right) \cdot e^{i\left[m \nu-2\left(\nu_{1}+\cdots+m \nu_{m}\right)\right] \theta}
$$

In particular,

$$
\lambda_{m}^{z, 1}[g(\theta)]=z \frac{\sin [(m+1) \theta]}{\sin \theta}
$$

which yields (6.11) after a simple computation with (6.10).

Noting that

$$
\sum_{\nu \geq 0} x^{\nu} \sum_{\substack{\vec{\nu} \in \mathbb{N}^{m+1} \\
|\vec{\nu}|=\nu}} \prod_{0 \leq j \leq m}\left(\begin{array}{c}
|z|+\nu_{j}-1 \\
\nu_{j}
\end{array}\right)=D\left(x, \operatorname{sym}^{m}[g(0)]\right)^{|z|}=(1-x)^{-(m+1)|z|}
$$

and comparing the coefficients of $x^{\nu}$, we obtain

$$
\sum_{\substack{\vec{\nu} \in \mathbb{N}^{m+1} \\
|\vec{\nu}|=\nu}} \prod_{0 \leq j \leq m}\left(\begin{array}{c}
|z|+\nu_{j}-1 \\
\nu_{j}
\end{array}\right)=\left(\begin{array}{c}
(m+1)|z|+\nu-1 \\
\nu
\end{array}\right) .
$$

Thus we deduce from (6.14) that for any $\theta \in \mathbb{R}$,

$$
\left|\lambda_{m}^{z, \nu}[g(\theta)]\right| \leq\left(\begin{array}{c}
(m+1)|z|+\nu-1 \\
\nu
\end{array}\right) .
$$

By using the Plancherel identity and the preceding inequality, we have

$$
\begin{aligned}
\sum_{0 \leq \nu^{\prime} \leq m \nu}\left|\mu_{m, \nu^{\prime}}^{z, \nu}\right|^{2} & =\frac{2}{\pi} \int_{0}^{\pi}\left|\lambda_{m}^{z, \nu}[g(\theta)] \sin \theta\right|^{2} \mathrm{~d} \theta \\
& \leq\left(\begin{array}{c}
(m+1)|z|+\nu-1 \\
\nu
\end{array}\right)^{2}
\end{aligned}
$$

which implies (6.12).

Lemma 6.2. Let $m, n \in \mathbb{N}$, let $2 \mid k$ and let $z \in \mathbb{C}$. Then we have

$$
\sum_{f \in \mathrm{H}_{k}^{*}(1)} \omega(f) \lambda_{\mathrm{sym}^{m} f}^{z}(n)=\lambda_{\mathrm{sym}^{m}}^{z}(n)+O_{m}\left(k^{-5 / 6} n^{m / 4} \log (2 n) r_{m}^{z}(n)\right),
$$


where $\lambda_{\mathrm{sym}^{m}}^{z}(n)$ and $r_{m}^{z}(n)$ are the multiplicative functions defined by

$$
\lambda_{\mathrm{sym}^{m}}^{z}\left(p^{\nu}\right):=\mu_{m, 0}^{z, \nu} \quad \text { and } \quad r_{m}^{z}\left(p^{\nu}\right):=(m \nu+1)\left(\begin{array}{c}
(m+1)|z|+\nu-1 \\
\nu
\end{array}\right),
$$

respectively. Further there is a constant $c=c(m)$ such that

$$
\sum_{n \leq t} r_{m}^{z}(n) \ll_{m} t[\log (e t)]^{z_{m}-1} e^{c|z| \log _{2}(|z|+3)}
$$

uniformly for $t \geq 1$ and $z \in \mathbb{C}$, where $z_{m}:=(m+1)^{2} z^{*}$ and $z^{*}$ is the smallest integer $n$ such that $n \geq|z|$.

Proof. Writing $n=p_{1}^{\nu_{1}} \cdots p_{r}^{\nu_{r}}$, the multiplicativity of $\lambda_{\mathrm{sym}^{m}}^{z}(n)$ and $\lambda_{f}(n)$ allows us to deduce

$$
\sum_{f \in \mathrm{H}_{k}^{*}(1)} \omega(f) \lambda_{\mathrm{sym}^{m} f}^{z}(n)=\sum_{\nu_{1}^{\prime}=0}^{m \nu_{1}} \cdots \sum_{\nu_{r}^{\prime}=0}^{m \nu_{r}} \prod_{j=1}^{r} \mu_{m, \nu_{j}^{\prime}}^{z, \nu_{j}} \sum_{f \in \mathrm{H}_{k}^{*}(1)} \omega(f) \lambda_{f}\left(p_{1}^{\nu_{1}^{\prime}} \cdots p_{r}^{\nu_{r}^{\prime}}\right) .
$$

We apply Corollary 2.2 in 8 with the choice $m=p_{1}^{\nu_{1}^{\prime}} \ldots p_{r}^{\nu_{r}^{\prime}}, n=1$ and $N=1$ there. The principal term contributes $\prod_{1 \leq j \leq r} \mu_{m, 0}^{z, \nu_{j}}=\lambda_{\mathrm{sym}^{m}}^{z}(n)$, and the error term constitutes a term

$$
\begin{aligned}
& \ll k^{-5 / 6} n^{m / 4} \log (2 n) \prod_{1 \leq j \leq r} \sum_{0 \leq \nu_{j}^{\prime} \leq m \nu_{j}}\left|\mu_{m, \nu_{j}^{\prime}}^{z, \nu_{j}}\right| \\
& \ll k^{-5 / 6} n^{m / 4} \log (2 n) r_{m}^{z}(n) .
\end{aligned}
$$

This proves (6.15). The estimate (6.17) is Lemma 4.3 of [21].

For notational convenience, we write

$$
\omega_{\mathrm{sym}^{m} f}^{z}(x):=\sum_{n=1}^{\infty} \frac{\lambda_{\mathrm{sym}^{m} f}^{z}(n)}{n} e^{-n / x} .
$$

The lemmas below are devoted to studying its average over all primitive forms, which leads to the integral formula in (6.20). This is a crucial step in the study of the moments, and is discovered in [1] with the insightful idea of the "equidistribution" properties of $g\left(\theta_{f}(p)\right)$ in (6.6).

Lemma 6.3. Let $m \in \mathbb{N}$, let $2 \mid k$, let $x \geq 3$ and let $z \in \mathbb{C}$. Then we have

$$
\begin{aligned}
\sum_{f \in \mathrm{H}_{k}^{*}(1)} \omega(f) \omega_{\mathrm{sym}^{m} f}^{z}(x)= & \sum_{n=1}^{\infty} \frac{\lambda_{\mathrm{sym}^{m}}^{z}(n)}{n} e^{-n / x} \\
& +O_{m}\left(k^{-5 / 6} x^{m / 4}\left[\left(z_{m}+1\right) \log x\right]^{z_{m}}\right) .
\end{aligned}
$$

Proof. By (6.18) and (6.15), we can write

$$
\begin{aligned}
\sum_{f \in \mathrm{H}_{k}^{*}(1)} \omega(f) \omega_{\mathrm{sym}^{m} f}^{z}(x)= & \sum_{n=1}^{\infty} \frac{\lambda_{\mathrm{sym}^{m}}^{z}(n)}{n} e^{-n / x} \\
& +O_{m}\left(\frac{1}{k^{5 / 6}} \sum_{n=1}^{\infty} \frac{\log (2 n)}{n^{1-m / 4}} e^{-n / x} r_{m}^{z}(n)\right)
\end{aligned}
$$


An integration by parts with (6.17) allows us to deduce

$$
\begin{aligned}
\sum_{n=1}^{\infty} \frac{\log (2 n)}{n^{1-m / 4}} e^{-n / x} r_{m}^{z}(n) & =\int_{1-}^{\infty} \frac{\log (2 t)}{t^{1-m / 4}} e^{-t / x} \mathrm{~d} \sum_{n \leq t} r_{m}^{z}(n) \\
& \ll_{m} e^{c|z| \log _{2}(|z|+3)} \int_{1}^{\infty} \frac{[\log (3 t)]^{z_{m}}}{t^{1-m / 4}} e^{-t / x}\left(1+\frac{t}{x}\right) \mathrm{d} t
\end{aligned}
$$

But we have

$$
\begin{aligned}
\int_{1}^{x} \frac{[\log (3 t)]^{z_{m}}}{t^{1-m / 4}} e^{-t / x}\left(1+\frac{t}{x}\right) \mathrm{d} t & \ll x^{m / 4}(\log x)^{z_{m}} \\
\int_{x}^{\infty} \frac{[\log (3 t)]^{z_{m}}}{t^{1-m / 4}} e^{-t / x}\left(1+\frac{t}{x}\right) \mathrm{d} t & \ll x^{m / 4} \int_{1}^{\infty} u^{m / 4} e^{-u}[\log (3 u x)]^{z_{m}} \mathrm{~d} u \\
& \ll x^{m / 4}(\log x)^{z_{m}} \sum_{\nu=0}^{z_{m}}\left(\begin{array}{c}
z_{m} \\
\nu
\end{array}\right) \int_{1}^{\infty} u^{m+\nu} e^{-u} \mathrm{~d} u \\
& \ll x^{m / 4}\left[\left(z_{m}+1\right) \log x\right]^{z_{m}}
\end{aligned}
$$

This completes the proof.

Lemma 6.4. Let $m \in \mathbb{N}$, let $z \in \mathbb{C}$ and let $z_{m}^{\prime}:=(m+1)|z|+3$. Then there is a positive constant $c=c(m)$ such that for any $\sigma \in\left(\frac{1}{2}, 1\right]$ we have

$$
\sum_{n \geq 1} \frac{\left|\lambda_{\mathrm{sym}^{m}}^{z}(n)\right|}{n^{\sigma}} \leq \exp \left\{c z_{m}^{\prime}\left(\log _{2} z_{m}^{\prime}+\frac{z_{m}^{\prime(1-\sigma) / \sigma}-1}{(1-\sigma) \log z_{m}^{\prime}}\right)\right\} .
$$

Further we have

$$
\sum_{n \geq 1} \frac{\lambda_{\mathrm{sym}^{m}}^{z}(n)}{n}=\prod_{p} \frac{2}{\pi} \int_{0}^{\pi} D\left(p^{-1}, \operatorname{sym}^{m}[g(\theta)]\right)^{z} \sin ^{2} \theta \mathrm{d} \theta .
$$

Proof. Using the multiplicativity of $\lambda_{\mathrm{sym}^{m}}^{z}(n)$ and (6.16), we obtain that for any $\sigma>\frac{1}{2}$

$$
\sum_{n \geq 1}\left|\lambda_{\mathrm{sym}^{m}}^{z}(n)\right| n^{-\sigma}=\prod_{p} \sum_{\nu \geq 0}\left|\mu_{m, 0}^{z, \nu}\right| p^{-\nu \sigma}
$$

From (6.11) and (6.12), we have

$$
\begin{aligned}
\sum_{\nu \geq 0} \frac{\left|\mu_{m, 0}^{z, \nu}\right|}{p^{\nu \sigma}} & \leq \sum_{\nu \geq 0} \frac{1}{p^{\nu \sigma}}\left(\begin{array}{c}
(m+1)|z|+\nu-1 \\
\nu
\end{array}\right)-\frac{(m+1)|z|}{p^{\sigma}} \\
& =\left(1-\frac{1}{p^{\sigma}}\right)^{-(m+1)|z|}-\frac{(m+1)|z|}{p^{\sigma}}
\end{aligned}
$$


Therefore, we deduce that by the estimate (3.20),

$$
\begin{aligned}
\prod_{p^{\sigma} \leq z_{m}^{\prime}} \sum_{\nu \geq 0} \frac{\left|\mu_{m, 0}^{z, \nu}\right|}{p^{\nu \sigma}} & \leq \prod_{p^{\sigma} \leq z_{m}^{\prime}}\left(1-\frac{1}{p^{\sigma}}\right)^{-(m+1)|z|} \\
& \leq \exp \left\{z_{m}\left(\sum_{p \leq z_{m}^{\prime 1 / \sigma}} p^{-\sigma}+O(1)\right)\right\} \\
& \leq \exp \left\{c z_{m}^{\prime}\left(\log _{2} z_{m}^{\prime}+\frac{z_{m}^{\prime(1-\sigma) / \sigma}-1}{(1-\sigma) \log z_{m}^{\prime}}\right)\right\}
\end{aligned}
$$

whereas for $p^{\sigma}>z_{m}^{\prime}$,

$$
\prod_{p^{\sigma}>z_{m}^{\prime}} \sum_{\nu \geq 0} \frac{\left|\mu_{m, 0}^{z, \nu}\right|}{p^{\nu \sigma}} \leq \exp \left\{\sum_{p^{\sigma}>z_{m}^{\prime}} \frac{c z_{m}^{\prime 2}}{p^{2 \sigma}}\right\} \leq e^{c z_{m}^{\prime 1 / \sigma} / \log z_{m}^{\prime}}
$$

via (6.22). (Note that the term for $\nu=1$ on the right of (6.22) vanishes.) Noting that

$$
\frac{z_{m}^{\prime 1 / \sigma}}{\log z_{m}^{\prime}}=z_{m}^{\prime} \frac{z_{m}^{\prime(1-\sigma) / \sigma}}{\log z_{m}^{\prime}} \leq z_{m}^{\prime} \frac{z_{m}^{\prime(1-\sigma) / \sigma}-1}{(1-\sigma) \log z_{m}^{\prime}}
$$

we obtain (6.19) by inserting (6.23) and (6.24) into (6.21).

The multiplicativity of $\lambda_{\operatorname{sym}^{m}}^{z}(n)$ and (6.10) imply

$$
\begin{aligned}
\sum_{n \geq 1} \frac{\lambda_{\mathrm{sym}^{m}}^{z}(n)}{n} & =\prod_{p} \sum_{\nu \geq 0} \frac{\mu_{m, 0}^{z, \nu}}{p^{\nu}} \\
& =\prod_{p} \frac{2}{\pi} \int_{0}^{\pi} \sum_{\nu \geq 0} \frac{\lambda_{m}^{z, \nu}[g(\theta)]}{p^{\nu}} \sin ^{2} \theta \mathrm{d} \theta \\
& =\prod_{p} \frac{2}{\pi} \int_{0}^{\pi} D\left(p^{-1}, \operatorname{sym}^{m}[g(\theta)]\right)^{z} \sin ^{2} \theta \mathrm{d} \theta
\end{aligned}
$$

by (6.2). This completes the proof.

Lemma 6.5. Let $m \in \mathbb{N}$, let $\sigma \in\left[0, \frac{1}{2}\right)$, let $x \geq 3$ and let $z \in \mathbb{C}$. There is a positive constant $c=c(m)$ such that

$$
\sum_{n=1}^{\infty} \frac{\lambda_{\mathrm{sym}^{m}}^{z}(n)}{n} e^{-n / x}=M_{\mathrm{sym}^{m}}^{z}+O_{m}\left(\frac{1}{x^{\sigma}} \exp \left\{c z_{m}^{\prime}\left(\log _{2} z_{m}^{\prime}+\frac{z_{m}^{\prime \sigma /(1-\sigma)}-1}{\sigma \log z_{m}^{\prime}}\right)\right\}\right) .
$$

The implied constant depends on $m$ only.

Proof. We first write

$$
\sum_{n=1}^{\infty} \frac{\lambda_{\mathrm{sym}^{m}}^{z}(n)}{n} e^{-n / x}=\sum_{n \geq 1} \frac{\lambda_{\mathrm{sym}^{m}}^{z}(n)}{n}+O\left(R_{1}+R_{2}\right),
$$

where

$$
R_{1}:=\sum_{n>x} \frac{\left|\lambda_{\mathrm{sym}^{m}}^{z}(n)\right|}{n}, \quad R_{2}:=\sum_{n \leq x} \frac{\left|\lambda_{\mathrm{sym}^{m}}^{z}(n)\right|}{n}\left|e^{-n / x}-1\right| .
$$

Clearly for any $\sigma \in\left[0, \frac{1}{2}\right)$, we have

$$
(n / x)^{\sigma} \gg \begin{cases}1 & \text { if } n>x, \\ \left|e^{-n / x}-1\right| & \text { if } n \leq x .\end{cases}
$$


Thus Lemma 6.4 implies

$$
\begin{aligned}
R_{1}+R_{2} & \ll \sum_{n \geq 1} \frac{\left|\lambda_{\mathrm{sym}^{m}}^{z}(n)\right|}{n}\left(\frac{n}{x}\right)^{\sigma} \\
& \ll x^{-\sigma} \exp \left\{c z_{m}^{\prime}\left(\log _{2} z_{m}^{\prime}+\frac{z_{m}^{\prime \sigma /(1-\sigma)}-1}{\sigma \log z_{m}^{\prime}}\right)\right\} .
\end{aligned}
$$

The proof is done.

Lemma 6.6. Let $\eta \in\left(0, \frac{1}{31}\right)$ fixed, let $m=1,2,3,4$, let $2 \mid k$ and let $f \in$ $\mathrm{H}_{k, \text { sym }}^{+}(1 ; \eta)$. Then we have

$$
L\left(1, \operatorname{sym}^{m} f\right)^{z}=\omega_{\operatorname{sym}^{m} f}^{z}(x)+O_{\eta}\left(\left(x^{-1 / \log _{2} k}+x^{c|z|} e^{-(\log k)^{2}}\right) e^{c|z| \log _{3}(8 k)}\right)
$$

uniformly for $2 \mid k, x \geq 3$ and $z \in \mathbb{C}$, where the positive constant $c=c(\eta)$ and the implied constant depend at most on $\eta$.

Proof. The method of proof is similar to that of Proposition 3.4. We express (6.18) with (4.3) and (6.7) into

$$
\omega_{\mathrm{sym}^{m} f}^{z}(x)=\frac{1}{2 \pi i} \int_{(1)} L\left(s+1, \operatorname{sym}^{m} f\right)^{z} \Gamma(s) x^{s} \mathrm{~d} s
$$

and shift the line of integration (1) to the path $\mathcal{C}$ consisting of straight lines joining

$$
\kappa_{1}-i \infty, \quad \kappa_{1}-i T, \quad-\kappa_{2}-i T, \quad-\kappa_{2}+i T, \quad \kappa_{1}+i T, \quad \kappa_{1}+i \infty,
$$

where $\kappa_{1}:=1 / \log x, \kappa_{2}:=1 / \log _{2} k$ and $T=(\log k)^{2}$. Therefore,

$$
\omega_{\text {sym }^{m} f}^{z}(x)=L\left(1, \operatorname{sym}^{m} f\right)^{z}+\frac{1}{2 \pi i} \int_{\mathcal{C}} L\left(s+1, \operatorname{sym}^{m} f\right)^{z} \Gamma(s) x^{s} \mathrm{~d} s .
$$

By Proposition 3.5 with the choice $\alpha=\kappa_{2}, \log L\left(s+1, \operatorname{sym}^{m} f\right) \ll_{\eta} \log _{3}(8 k)$ for all $s \in \mathcal{C}$ with $|\tau| \leq 4 T$, and by (3.10), $\log L\left(s+1, \operatorname{sym}^{m} f\right) \ll \log x$ for $\Re e s=\kappa_{1}$. It follows that

$$
\begin{aligned}
\frac{1}{2 \pi i} \int_{\mathcal{C}} L\left(s+1, \operatorname{sym}^{m} f\right)^{z} \Gamma(s) x^{s} \mathrm{~d} s \ll_{\eta} & x^{-\kappa_{2}} e^{c|z| \log _{3} k} \int_{|y| \leq T}\left|\Gamma\left(1-\kappa_{2}+i y\right)\right| \mathrm{d} y \\
& +e^{c|z| \log _{3} k} \int_{-\kappa_{2}}^{\kappa_{1}}|\Gamma(1+\alpha+i T)| \mathrm{d} \alpha \\
& +e^{c|z| \log x} \int_{|y| \geq T}\left|\Gamma\left(1+\kappa_{1}+i y\right)\right| \mathrm{d} y .
\end{aligned}
$$

The proof is then complete with (3.17).

Now we are ready to prove Proposition 6.1. We deduce from Lemma 6.6 that

$$
\sum_{f \in \mathrm{H}_{k, \mathrm{sym}}^{+} m(1 ; \eta)} \omega(f) L\left(1, \operatorname{sym}^{m} f\right)^{z}=\sum_{f \in \mathrm{H}_{k, \mathrm{sym}}^{+} m(1 ; \eta)} \omega(f) \omega_{\mathrm{sym}^{m} f}^{z}(x)+O_{\eta}\left(R_{1}\right),
$$

where for $c=c(\eta)$ a positive constant

$$
R_{1}:=\left(x^{-1 / \log _{2} k}+x^{c|z|} e^{-(\log k)^{2}}\right) e^{c|z| \log _{3}(8 k)} .
$$


Here we have used

$$
\sum_{f \in \mathrm{H}_{k, \mathrm{sym}}^{+} m} \omega(1 ; \eta) \leq \sum_{f \in \mathrm{H}_{k}^{*}(1)} \omega(f)=1+O\left(k^{-5 / 6}\right) .
$$

On the other hand, for $\varepsilon>0, f \in \mathrm{H}_{k}^{*}(1), x \geq 3$ and $z \in \mathbb{C}$, we have

$$
\omega_{\mathrm{sym}^{m} f}^{z}(x)=\frac{1}{2 \pi i} \int_{(\varepsilon)} L\left(s+1, \operatorname{sym}^{m} f\right)^{z} \Gamma(s) x^{s} \mathrm{~d} s \ll \iota(\varepsilon)^{|\Re e z|} x^{\varepsilon},
$$

where $\iota(\varepsilon)=\zeta(1+\varepsilon)^{m+1}>0$ is a constant depending on $\varepsilon$. Together with (1.11), we see that

$$
\left|\sum_{f \in \mathrm{H}_{k, \mathrm{sym}^{m}}^{-}(1 ; \eta)} \omega(f) \omega_{\mathrm{sym}^{m} f}^{z}(x)\right| \ll_{\eta} \iota(\varepsilon)^{|\Re e z|} x^{\varepsilon} k^{31 \eta-1} .
$$

Hence we input the forms of $\mathrm{H}_{k, \mathrm{sym}^{m}}^{-}(1 ; \eta)$ into (6.21) with a negligible additional error to get

$$
\sum_{f \in \mathrm{H}_{k, \mathrm{sym}}^{+} m} \omega(1 ; \eta) L\left(1, \operatorname{sym}^{m} f\right)^{z}=\sum_{f \in \mathrm{H}_{k}^{*}(1)} \omega(f) \omega_{\mathrm{sym}^{m} f}^{z}(x)+O_{\eta}\left(R_{2}\right),
$$

where $R_{2}:=R_{1}+x^{\varepsilon} k^{31 \eta-1} \iota(\varepsilon)^{|z|}$. Finally by using Lemmas 6.3 and 6.5 , we have

$$
\sum_{f \in \mathrm{H}_{k, \mathrm{sym}}^{+} m(1 ; \eta)} \omega(f) L\left(1, \operatorname{sym}^{m} f\right)^{z}=M_{\mathrm{sym}^{m}}^{z}+O_{\eta}\left(R_{3}\right),
$$

where

$$
\begin{aligned}
R_{3}:=( & \left.x^{-1 / \log _{2} k}+x^{c|z|} e^{-(\log k)^{2}}\right) e^{c|z| \log _{3}(8 k)}+k^{-(1-31 \eta)} x^{\varepsilon} \iota(\varepsilon)^{|z|} \\
& +\frac{x^{m / 4}}{k^{5 / 6}}\left[\left(z_{m}+1\right) \log x\right]^{z_{m}}+\frac{1}{x^{\sigma}} \exp \left\{c z_{m}^{\prime}\left(\log _{2} z_{m}^{\prime}+\frac{z_{m}^{\prime \sigma /(1-\sigma)}-1}{\sigma \log z_{m}^{\prime}}\right)\right\} .
\end{aligned}
$$

Now taking $\varepsilon=\frac{1}{500 m}, x^{m}=k^{1 / 10}$ and $\sigma=1 / \log (|z|+8)$, it is easy to verify that there are two positive constants $c$ and $\delta$ depending at most on $\eta$ such that

$$
R_{3} \ll e^{-\delta \log k / \log _{2} k}
$$

uniformly for $2 \mid k$ and $|z| \leq c \log k / \log _{2}(8 k) \log _{3}(8 k)$. This proves the desired asymptotic formula.

Finally by using Proposition 3.2 (note $L\left(1, \mathrm{sym}^{m} f\right)>0$ ) and (1.11), for $m=$ $1,2,3,4$ if $\Re e z \geq 0$ or $m=1,2,4$ if $\Re e z<0$, we have

$$
\sum_{f \in \mathrm{H}_{k, \mathrm{sym}^{-}}^{-}(1 ; \eta)} \omega(f) L\left(1, \operatorname{sym}^{m} f\right)^{z} \ll_{\eta} k^{31 \eta-1}(\log k)^{c} .
$$

Hence the input of these forms into (6.28) causes a tolerable error there. The same choice of parameters allows us to obtain the required result.

\section{Proof of Theorem 2}

From Proposition 6.1, we have

$$
\frac{1}{2} M_{\mathrm{sym}^{m}}^{ \pm r} \leq \sum_{f \in \mathrm{H}_{k, \mathrm{sym} m}^{+}(1 ; \eta)} \omega(f) L\left(1, \operatorname{sym}^{m} f\right)^{ \pm r}
$$


for $0<r \leq c \log k / \log _{2}(8 k) \log _{3}(8 k)$ and all sufficiently large even integer $k$. In view of (6.27), there are $f_{m}^{ \pm} \in \mathrm{H}_{k, \text { sym }^{m}}^{+}(1 ; \eta)$ such that

$$
\frac{1}{2} M_{\mathrm{sym}^{m}}^{ \pm r} \leq 2 L\left(1, \operatorname{sym}^{m} f_{m}^{ \pm}\right)^{ \pm r} .
$$

According to [1] and 21], we have

$$
\log M_{\mathrm{sym}^{m}}^{ \pm r}=A_{m}^{ \pm} r \log \left(B_{m}^{ \pm} \log r\right)+O_{m}\left(\frac{r}{\log r}\right)
$$

where

$$
\left\{\begin{array}{l}
A_{m}^{ \pm}:=\max _{\theta \in[0, \pi]} \pm \operatorname{tr}\left(\operatorname{sym}^{m}[g(\theta)]\right)= \pm \operatorname{tr}\left(\operatorname{sym}^{m}\left[g\left(\theta_{m}^{ \pm}\right)\right]\right) \\
B_{m}^{ \pm}:=\exp \left\{\gamma_{0}+\frac{1}{A_{m}^{ \pm}} \sum_{p}\left( \pm \log D\left(p^{-1}, \operatorname{sym}^{m}\left[g\left(\theta_{m, p}^{ \pm}\right)\right]\right)-\frac{A_{m}^{ \pm}}{p}\right)\right\} .
\end{array}\right.
$$

Here $\gamma_{0}$ is a constant determined by

$$
\sum_{p \leq t} \frac{1}{p}=\log _{2} t+\gamma_{0}+O\left(\frac{1}{\log t}\right)
$$

and $\theta_{m, p}^{ \pm} \in[0, \pi]$ are real numbers such that

$$
\left\{\begin{array}{l}
D\left(p^{-1}, \operatorname{sym}^{m}\left[g\left(\theta_{m, p}^{+}\right)\right]\right)=\max _{\theta \in[0, \pi]} D\left(p^{-1}, \operatorname{sym}^{m}[g(\theta)]\right), \\
D\left(p^{-1}, \operatorname{sym}^{m}\left[g\left(\theta_{m, p}^{-}\right)\right]\right)=\min _{\theta \in[0, \pi]} D\left(p^{-1}, \operatorname{sym}^{m}[g(\theta)]\right) .
\end{array}\right.
$$

The choice $r=c \log k / \log _{2}(8 k) \log _{3}(8 k)$ in (7.1) gives the required result.

In view of (6.1), the constants $A_{m}^{ \pm}, B_{m}^{ \pm}, \theta_{m}^{ \pm}$and $\theta_{m, p}^{ \pm}$are indeed defined for all $m \in \mathbb{N}$. For completeness, we give a general discussion. It is easy to see that for all primes $p, \theta_{m}^{+}=\theta_{m, p}^{+}=0$ for $m \in \mathbb{N}, \theta_{m}^{-}=\theta_{m, p}^{-}=\pi$ for $2 \nmid m$ and $\theta_{2}^{-}=\theta_{2, p}^{-}=\frac{1}{2} \pi$. These give the listed values for $A_{m}^{ \pm}$and $B_{m}^{ \pm}$in (1.15) (see [5], [19] and [1] for the computation).

When $m \geq 4$ and $2 \mid m$, a simple expression for $B_{m}^{-}$does not seem available. We only give the detailed computation for $m=4$. It is plain to see that $A_{4}^{-}=$ $-\operatorname{tr}\left(\operatorname{sym}^{4}\left[g\left(\theta_{4}^{-}\right)\right]\right)=\frac{5}{4}$, where $\theta_{4}^{-} \in[0, \pi]$ satisfies $\cos \theta_{4}^{-}=-\frac{1}{4}$.

Clearly we have

$$
D\left(p^{-1}, \operatorname{sym}^{4}\left[g\left(\theta_{4, p}^{-}\right)\right]\right)^{-1}=\max _{\theta \in[0, \pi]} D\left(p^{-1}, \operatorname{sym}^{4}[g(\theta)]\right)^{-1} .
$$

A simple calculation shows that

$$
D\left(p^{-1}, \operatorname{sym}^{4}[g(\theta)]\right)^{-1}=\left(1-p^{-1}\right)\left\{\left(1+p^{-2}\right)^{2}+2 p^{-1} h_{p}(\cos \theta)\right\},
$$

where

$$
h_{p}(t):=4 p^{-1} t^{3}-2\left(1+p^{-2}\right) t^{2}-\left(1+p^{-1}\right)^{2} t+1+p^{-2} .
$$

It is then easy to show that $\theta_{4, p}^{-} \in[0, \pi]$ is determined by

$$
\cos \theta_{4, p}^{-}=\left(1+p^{-2}-\sqrt{1+3 p^{-1}+8 p^{-2}+3 p^{-3}+p^{-4}}\right) /\left(6 p^{-1}\right) .
$$

From these formulas, an elementary calculation leads to (1.16). This completes the proof. 


\section{Proof of Theorem 3}

The proofs for parts (i) and (ii) are essentially the same, both relying on Proposition 3.4, except for different choices of parameters: $s=1$ and $T=(\log k)^{4 / \eta}$ without GRH (or $s=1, \alpha=\frac{3}{4}$ and $T=(\log k)^{2+20 \varepsilon}$ under GRH). Then for $f \in \mathrm{H}_{k, \text { sym }^{m}}^{+}(1 ; \eta)$ where $\eta \in\left(0, \frac{1}{31}\right)$ is a fixed constant (or any $f \in \mathrm{H}_{k}^{*}(1)$ under $\mathrm{GRH})$, we derive that

$$
\log L\left(1, \operatorname{sym}^{m} f\right)=\sum_{n=2}^{\infty} \frac{\Lambda_{\mathrm{sym}^{m} f}(n)}{n \log n} e^{-n / T}+o(1) .
$$

With the trivial estimate for (3.7), Lebesgue's dominated convergence theorem implies that

$$
\sum_{p} \sum_{\nu \geq 2} \frac{\Lambda_{\mathrm{sym}^{m} f}\left(p^{\nu}\right)}{p^{\nu} \log p^{\nu}}\left(e^{-p^{\nu} / T}-e^{-\nu p / T}\right) \rightarrow 0 \quad(k \rightarrow \infty) .
$$

Thus we manipulate with (3.7) as follows:

$$
\begin{aligned}
\sum_{n=2}^{\infty} \frac{\Lambda_{\mathrm{sym}^{m} f}(n)}{n \log n} e^{-n / T} & =\sum_{p} \sum_{\nu \geq 1} \frac{\Lambda_{\mathrm{sym}^{m} f}\left(p^{\nu}\right)}{p^{\nu} \log p^{\nu}} e^{-p^{\nu} / T} \\
& =\sum_{p} \sum_{\nu \geq 1} \frac{\Lambda_{\mathrm{sym}^{m} f}\left(p^{\nu}\right)}{p^{\nu} \log p^{\nu}} e^{-\nu p / T}+o(1) \\
& =\sum_{p} \sum_{\nu \geq 1} \sum_{0 \leq j \leq m} \frac{\alpha_{f}(p)^{(m-2 j) \nu}}{\nu p^{\nu}} e^{-\nu p / T}+o(1) \\
& =\sum_{p} \sum_{0 \leq j \leq m} \log \left(1-\frac{\alpha_{f}(p)^{m-2 j}}{e^{p / T} p}\right)^{-1}+o(1) \\
& =\sum_{p} \log D\left(e^{-p / T} p^{-1}, \operatorname{sym}^{m}\left[g\left(\theta_{f}(p)\right)\right]\right)+o(1),
\end{aligned}
$$

by (6.1), where $\theta_{f}(p) \in[0, \pi]$ such that $\alpha_{f}(p)=e^{i \theta_{f}(p)}$.

On one hand, we have

$$
\left|\sum_{p>T} \log D\left(e^{-p / T} p^{-1}, \operatorname{sym}^{m}\left[g\left(\theta_{f}(p)\right)\right]\right)\right| \ll \sum_{p>T} \frac{e^{-p / T}}{p} \ll \frac{1}{\log T} \rightarrow 0
$$

and on the other hand,

$$
\left|\sum_{p \leq T} \log \left(\frac{D\left(e^{-p / T} p^{-1}, \operatorname{sym}^{m}\left[g\left(\theta_{f}(p)\right)\right]\right)}{D\left(p^{-1}, \operatorname{sym}^{m}\left[g\left(\theta_{f}(p)\right)\right]\right)}\right)\right| \ll \sum_{p \leq T} \frac{1-e^{-p / T}}{p} \ll \frac{1}{\log T} \rightarrow 0 .
$$

Inserting these relations into (8.1), we get

$$
\log L\left(1, \operatorname{sym}^{m} f\right)=\sum_{p \leq T} \log D\left(p^{-1}, \operatorname{sym}^{m}\left[g\left(\theta_{f}(p)\right)\right]\right)+o(1) .
$$


Thus the definition of $\theta_{m, p}^{ \pm}($see $(7.5)$ ) allows us to write

$$
\begin{aligned}
\sum_{p \leq T} \log D\left(p^{-1}, \operatorname{sym}^{m}\left[g\left(\theta_{m, p}^{+}\right)\right]\right)+o(1) & \geq \log L\left(1, \operatorname{sym}^{m} f\right) \\
& \geq \sum_{p \leq T} \log D\left(p^{-1}, \operatorname{sym}^{m}\left[g\left(\theta_{m, p}^{-}\right)\right]\right)+o(1) .
\end{aligned}
$$

By (6.4) and (7.3), we have

$$
\begin{aligned}
0 & \leq \mp \log \left(\frac{D\left(p^{-1}, \operatorname{sym}^{m}\left[g\left(\theta_{m}^{ \pm}\right)\right]\right)}{D\left(p^{-1}, \operatorname{sym}^{m}\left[g\left(\theta_{m, p}^{ \pm}\right)\right]\right)}\right) \\
& =\mp \frac{ \pm A_{m}^{ \pm}-\operatorname{tr}\left(\operatorname{sym}^{m}\left[g\left(\theta_{m, p}^{ \pm}\right)\right]\right)}{p}+O\left(\frac{1}{p^{2}}\right) . \\
& =-\frac{A_{m}^{ \pm} \mp \operatorname{tr}\left(\operatorname{sym}^{m}\left[g\left(\theta_{m, p}^{ \pm}\right)\right]\right)}{p}+O\left(\frac{1}{p^{2}}\right) .
\end{aligned}
$$

Since $A_{m}^{ \pm} \mp \operatorname{tr}\left(\operatorname{sym}^{m}\left[g\left(\theta_{m, p}^{ \pm}\right)\right]\right) \geq 0$, it follows that

$$
\frac{A_{m}^{ \pm} \mp \operatorname{tr}\left(\operatorname{sym}^{m}\left[g\left(\theta_{m, p}^{ \pm}\right)\right]\right)}{p} \ll \frac{1}{p^{2}} .
$$

On the other hand, we have

$$
\log D\left(p^{-1}, \operatorname{sym}^{m}\left[g\left(\theta_{m, p}^{ \pm}\right)\right]\right)-\operatorname{tr}\left(\operatorname{sym}^{m}\left[g\left(\theta_{m, p}^{ \pm}\right)\right]\right) / p \ll 1 / p^{2}
$$

by (6.4) again. From (8.4) and (8.5), we deduce that

$$
\pm \log D\left(p^{-1}, \operatorname{sym}^{m}\left[g\left(\theta_{m, p}^{ \pm}\right)\right]\right)-A_{m}^{ \pm} / p \ll 1 / p^{2}
$$

and hence,

$$
\sum_{p>T}\left( \pm \log D\left(p^{-1}, \operatorname{sym}^{m}\left[g\left(\theta_{m, p}^{ \pm}\right)\right]\right)-\frac{A_{m}^{ \pm}}{p}\right) \ll \frac{1}{T \log T}
$$

Combining these two, we infer that

$$
\begin{aligned}
& \sum_{p \leq T} \log D\left(p^{-1}, \operatorname{sym}^{m}\left[g\left(\theta_{m, p}^{ \pm}\right)\right]\right) \\
& \quad= \pm \sum_{p \leq T} \frac{A_{m}^{ \pm}}{p} \pm \sum_{p}\left( \pm \log D\left(p^{-1}, \operatorname{sym}^{m}\left[g\left(\theta_{m, p}^{ \pm}\right)\right]\right)-\frac{A_{m}^{ \pm}}{p}\right)+O\left(\frac{1}{T \log T}\right) \\
& \quad= \pm A_{m}^{ \pm} \log \left(B_{m}^{ \pm} \log T\right)+O\left(\frac{1}{T \log T}\right)
\end{aligned}
$$

with (7.3). Inserting it into (8.2), we obtain (1.17) and (1.18) accordingly, in view of the parameters chosen at the beginning of the proof. We also remark that the constant 2 before $B_{m}^{ \pm}$in (1.18) comes from the factor 2 in the exponent of $\log k$ in (3.15). 


\section{Proofs of Theorems 4 And 5}

According to (8.2), for $m=1,2,3,4,2 \mid k, T=(\log k)^{4 / \eta}$ and $f \in \mathrm{H}_{k, \mathrm{sym}^{m}}^{+}(1 ; \eta)$ where $\eta \in\left(0, \frac{1}{31}\right)$ is a fixed constant, we have by (8.2), (6.4) and (6.5),

$$
\begin{aligned}
\log L\left(1, \operatorname{sym}^{m} f\right) & =\sum_{p \leq T} \log D\left(p^{-1}, \operatorname{sym}^{m}\left[g\left(\theta_{f}(p)\right)\right]\right)+o(1) \\
& =\sum_{p \leq T} \frac{\lambda_{m}^{1,1}\left[g\left(\theta_{f}(p)\right)\right]}{p}+O_{\eta}(1) \\
& =\sum_{p \leq T} \frac{\lambda_{f}\left(p^{m}\right)}{p}+O(1) .
\end{aligned}
$$

By using (1.14) of Theorem 2, there is $f_{m}^{-} \in \mathrm{H}_{k, \mathrm{sym}^{m}}^{+}(1 ; \eta)$ such that

$$
\sum_{p \leq T} \frac{\lambda_{f_{m}^{-}}\left(p^{m}\right)}{p}=\log L\left(1, \operatorname{sym}^{m} f_{m}^{-}\right)+O(1) \leq-A_{m}^{-} \log _{3} k+O(1) .
$$

From (6.8) and (7.3), $\lambda_{f_{m}^{-}}\left(p^{m}\right)+A_{m}^{-} \geq 0$. As $\sum_{p \leq T} p^{-1}=\log _{3} k+O(1)$, we obtain by (9.1) that

$$
0 \leq \sum_{p \leq T} \frac{\lambda_{f_{m}^{-}}\left(p^{m}\right)+A_{m}^{-}}{p} \ll 1
$$

Therefore, for any function $\xi(k) \rightarrow \infty(k \rightarrow \infty)$ satisfying $\xi(k) \leq \log _{3} k$, we have

$$
\sum_{\substack{p \leq T \\ \lambda_{f_{m}^{-}}\left(p^{m}\right) \geq-A_{m}^{-}+\xi(k) / \log _{3} k}} \frac{1}{p} \leq \frac{\log _{3} k}{\xi(k)} \sum_{p \leq T} \frac{A_{m}^{-}+\lambda_{f_{m}^{-}}\left(p^{m}\right)}{p} \ll \frac{\log _{3} k}{\xi(k)},
$$

whence

$$
\begin{aligned}
\sum_{\substack{p \leq T \\
\lambda_{f_{m}^{-}}\left(p^{m}\right)<-A_{m}^{-}+\xi(k) / \log _{3} k}} \frac{1}{p} & =\sum_{p \leq T} \frac{1}{p}-\sum_{\substack{\lambda_{f_{m}^{-}}\left(p^{m}\right) \geq-A_{m}^{-}+\xi(k) / \log _{3} k \\
p}} \frac{1}{p} \\
& =\log _{3} k+O_{\eta}\left(\frac{\log _{3} k}{\xi(k)}\right) .
\end{aligned}
$$

Our result

$$
\sum_{\substack{p \leq(\log k)^{A} \\ \lambda_{f_{m}^{-}}\left(p^{m}\right)<-A_{m}^{-}+\xi(k) / \log _{3} k}} \frac{1}{p}=\log _{3} k+O_{A, \eta}\left(\frac{\log _{3} k}{\xi(k)}\right)
$$

follows, since

$$
\sum_{T<p \leq(\log k)^{A}} \frac{1}{p} \ll_{A, \eta} 1 .
$$

Taking $m=1$ and $m=2$ give (1.24) and (1.25), but for the latter result (1.25), we need the observation $\lambda_{f}(p)^{2}=\lambda_{f}\left(p^{2}\right)+1$ and $A_{2}^{-}=1$. In other words, $\lambda_{f}(p)^{2}=$ $\lambda_{f}\left(p^{2}\right)+A_{2}^{-}$.

To prove (1.23), it suffices to replace $f_{m}^{-}$by $f_{m}^{+}$and reverse the corresponding inequalities. 


\section{ACKNOWLEDGEMENT}

We are greatly indebted to the referee for many valuable suggestions, including the mollification process in Section 5 which simplifies much of our previous argument, and the insight in removing GRH in Theorems 4 and 5 of the early version. We express our sincere gratitude to Professors J. Cogdell and P. Michel for their paper 1, and for their kind help in our study of their work and the archimedean factor of the Rankin-Selberg $L$-function. We also thank Professor E. Kowalski for his reading and comments on an earlier form of this paper.

\section{REFERENCES}

1. J. Cogdell \& P. Michel, On the complex moments of symmetric power $L$-functions at $s=1$, IMRN 31 (2004), 1561-1618. MR2035301

2. S. Gelbart \& H. Jacquet, A relation between automorphic representations of GL(2) and GL(3), Ann. Sci. École Norm. Sup. (4) 11 (1978), 471-552. MR0533066 (81e:10025)

3. D. Goldfeld, J. Hoffstein \& D. Lieman, An effective zero-free region, Ann. of Math. (2) 140 (1994), no. 1, 177-181, appendix of [6]. MR1289494 (95m:11048)

4. A. Granville \& K. Soundararajan, Large character sums, J. Amer. Math. Soc. 14 (2001), 365-397. MR1815216 (2002h:11074)

5. L. Habsieger \& E. Royer, $L$-functions of automorphic forms and combinatorics: Dyck paths, Ann. Inst. Fourier (Grenoble), to appear.

6. J. Hoffstein \& P. Lockhart, Coefficients of Maass forms and the Siegel zero, Annals of Math. 140 (1994), 161-181. MR1289494 (95m:11048)

7. H. Iwaniec, Topics in Classical Automorphic Forms, Graduate Studies in Mathematics, vol. 17, American Mathematical Society, Providence, Rhode Island, 1997. MR1474964(98e:11051)

8. H. Iwaniec, W. Luo \& P. Sarnak, Low lying zeros of families of $L$-functions, Inst. Hautes Études Sci. Publ. Math. 91 (2000), 55-131. MR.1828743(2002h:11081)

9. H. Kim, Functoriality for the exterior square of $G L_{4}$ and symmetric fourth of $G L_{2}$, Appendix 1 by Dinakar Ramakrishnan, Appendix 2 by Henry H. Kim and Peter Sarnak, J. Amer. Math. Soc. 16 (2003), 139-183. MR1937203 (2003k:11083)

10. H. Kim \& F. Shahidi, Functorial products for $G L_{2} \times G L_{3}$ and functorial symmetric cube for $G L_{2}$ (with an appendix by C.J. Bushnell and G. Henniart), Ann. of Math. 155 (2002), 837-893. MR1923967 (2003m:11075)

11. H. Kim \& F. Shahidi, Cuspidality of symmetric power with applications, Duke Math. J. 112 (2002), 177-197. MR 1890650 (2003a:11057)

12. A.W. Knapp, Local Langlands correspondence: the Archimedean case, Proc. Sympos. Pure Math. 55 (1994), Part 2, 393-410. MR1265560 (95d:11066)

13. E. Kowalski \& P. Michel, Zeros of families of automorphic $L$-functions close to 1 and applications, Pacific J. Math. 207 (2002), no. 2, p. 411-431. MR:1972253(2004e:11047)

14. D.H. Lehmer, Some functions of Ramanujan, Math. Student 27 (1959), 105-116. MR0131412 (24:A1263)

15. W. Luo, Values of symmetric square $L$-functions at 1, J. Reine Angew. Math. 506 (1999), 215-235. MR1665705 (2001d:11055)

16. H.L. Montgomery, Topics in multiplicative number theory, Lecture Notes in Mathematics 227, Springer-Verlag, Bermion-Heidelberg-New York, 1971. MR0337847 (49:2616)

17. R.A. Rankin, An $\Omega$-result for the coefficients of cusp forms, Math. Ann. 283 (1973), 239-250. MR0321876 (48:241)

18. E. Royer, Statistique de la variable aléatoire $L\left(1, \mathrm{sym}^{2} f\right)$, Math. Ann. 321 (2001), 667-687. MR:1871974 (2003c:11045)

19. E. Royer, Interprétation combinatoire des moments négatifs des valeurs de fonctions $L$ au bord de la bande critique, Ann. Sci. École Norm. Sup. (4) 36 (2003), 601-620. MR 2013928 (2004k:11078)

20. E. Royer \& J. Wu, Taille des valeurs de fonctions $L$ de carrés symétriques au bord de la bande critique, Revista Matemática Iberoamericana 21 (2005), 263-312.

21. E. Royer \& J. Wu, Central values, values at the edge of the critical strip of symmetric power $L$-functions and Hecke eigenvalues, preprint. 
22. Z. Rudnick \& P. Sarnak, Zeros of principal $L$-functions and random matrix theory, Duke Math. J. 81 (1996), 269-322. MR1395406 (97f:11074)

23. J.-P. Serre, Quelques applications du théorème de densité de Chebotarev, Inst. Hautes Études Sci. Publ. Math. 54 (1981), 323-401. MR0644559 (83k:12011)

24. J.-P. Serre, Répartition asymptotique des valeurs propres de l'opérateur de Hecke $T_{p}, J$. Amer. Math. Soc. 10 (1997), 75-102. MR1396897(97h:11048)

25. G. Tenenbaum \& J. Wu, Moyennes de certaines fonctions multiplicatives sur les entiers friables, J. Reine Angew. Math. 564 (2003), 119-167. MR2021037 (2004m:11151)

26. E.C. Titchmarsh, The theory of function, Second edition, Oxford University Press, Oxford, 1952. MR0197687 (33:5850)

27. E.C. Titchmarsh, The theory of the Riemann zeta-function, 2nd edition revised by D.R. Heath-Brown, Oxford. MR0882550 (88c:11049)

Department of Mathematics, The University of Hong Kong, Pokfulam Road, Hong KONG

E-mail address: yklau@maths.hku.hk

Institut Elie Cartan, UMR 7502 UHP-CNRS-INRIA, Université Henri Poincaré, 54506 VANDGuVRe-LÈs-NANCY, FranCE

E-mail address: wujie@iecn.u-nancy.fr 